\title{
The Global Prevalence of Depression, Anxiety, Stress, and Insomnia Among General Population During COVID-19 Pandemic: A Systematic Review and Meta-analysis
}

\author{
Sultan Mahmud ${ }^{1}$ D $\cdot$ Md Mohsin $^{1} \cdot$ Md. Nayem Dewan ${ }^{1} \cdot$ Abdul Muyeed $^{2}$
}

Accepted: 18 October 2021 / Published online: 4 January 2022

(c) Associação Brasileira de Psicologia 2021

\begin{abstract}
This study aimed to examine research findings related to depression, anxiety, stress, and insomnia during the COVID-19 pandemic. This study also explored periodic changes in the prevalence of depression, anxiety, stress, and insomnia among the general people during this pandemic. We performed a meta-analysis by searching articles from several sources (PubMed, MEDLINE, and Google Scholar). We used the random-effects models, subgroup analysis, and heterogeneity test approaches. Results show that the prevalence of depression, stress, and insomnia increased during March to April 2020 (30.51\%, 29.4\%, and 25\%, respectively) compared to the study period before February $2020(25.25 \%, 16.27 \%$, and $22.63 \%$, respectively) and followed in May to June 2020 (16.47\%, 5.1\%, and 19.86, respectively). The prevalence of depression and anxiety from $k=30$ studies was $28.18 \%$ (95\% CI: $23.81-$ 32.54) and 29.57\% (95\% CI: 24.67-34.47), respectively. And the prevalence of stress $(k=13)$ was $25.18 \%$ (95\% CI: $14.82-35.54)$, and the prevalence of insomnia $(k=12)$ was $23.50 \%$ (95\% CI: 16.44-30.57). These prevalence estimates during the pandemic are very high compared to normal times. Hence, the governments and policymakers should apply proven strategies and interventions to avoid psychological adversity and improve overall mental health during the COVID-19 pandemic.
\end{abstract}

Keywords COVID-19 · Meta-analysis · Depression · Anxiety · Stress · Insomnia · General population

Sultan Mahmud

smahmud@isrt.ac.bd

1 Department of Applied Statistics, Institute of Statistical Research and Training, University of Dhaka, Dhaka 1000, Bangladesh

2 Department of Statistics, Jatiya Kabi Kazi Nazrul Islam University, Trishal, Mymensingh 2224, Bangladesh 


\section{Introduction}

In December 2019, a highly infectious acute respiratory syndrome caused by a novel coronavirus (SARS-CoV-2) originated in the city of Wuhan, China. The World Health Organization (WHO), on March 11, 2020, declared COVID-19 (the disease caused by the coronavirus) a pandemic (Huang et al., 2020; Mahmud et al., 2021b, 2021c; Mahmud et al., 2021a). It has already claimed several millions of lives across the globe. Its impact, however, should be assessed not only in terms of biological outcomes, but also in terms of economic, health, psychological, and social implications (O'Connor et al., 2020). It is normal to find higher psychological morbidities in the population in the event of a pandemic situation due to the widespread prevalence of disease and the increased number of cases and deaths (Krishnamoorthy et al., 2020). During outbreaks of the severe acute respiratory syndrome (SARS), H1N1 influenza, Ebola virus, and Middle East respiratory syndrome (MERS), related cases of higher psychological morbidities were also found in the past (Brooks et al., 2020). At least one of the many psychiatric morbidities such as depression, anxiety, stress, or sleep disorders occurred in over half of the patients with SARS, MERS, or Ebola (Chua et al., 2004; Jeong et al., 2016; Keita et al., 2017). As a ubiquitous infectious disease, COVID-19 may also affect the health, safety, and well-being of both individuals and community levels that are correlated with psychological distress and symptoms of mental illness (Bao et al., 2020). A recent study indicates that isolated and quarantined people go through substantial levels of anxiety, anger, confusion, and stress (Brooks et al., 2020). Due to the highly infectious and lethal nature of the virus, COVID-19 may disturb the mental health of people globally from infected patients, and healthcare workers to families, children, and students (Ryu et al., 2020; Bao et al., 2020; Chen et al., 2020). The pandemic has created enormous stress and fears, especially among elderly people due to their weak immune systems and chronic underlying diseases (Chen et al., 2020; Meng et al., 2020). Sometimes, psychological issues go unnoticed, especially during a pandemic due to the more direct impact of morbidity caused by a disease. But it is crucial to investigate the adverse psychosocial effects during long-term disasters like the COVID-19 pandemic in order to aid immediate and long-term recovery (O'Connor et al., 2020). Also, it is important to have a global view of the mental health problems and their impacts during the ongoing pandemic, because it may help define more effective strategies to fight off psychological problems during the COVID-19 pandemic and thereafter. Therefore, it is a pressing need to quantify the extent of psychological threats the COVID-19 pandemic places on people throughout the world.

Few published systematic reviews have been found on the same topic. A systematic review and meta-analysis (Salari et al., 2020) of 17 studies showed that the prevalence of depression, anxiety, and stress among the general population was respectively $33.7 \%, 31.9 \%$, and $29.6 \%$. Those 17 studies were published before May 2020. Another meta-analysis (Cooke et al., 2020) considered 14 studies published before May 26, 2020, that displayed only the prevalence 
of posttraumatic and psychological stress among the general population during the COVID-19 pandemic. The study found that the prevalence of posttraumatic and psychological stress among the general population was $23.88 \%$ and $24.84 \%$, respectively. Three similar types of meta-analysis (da Silva \& Neto 2020; Pappa et al., 2020; Mahmud et al., 2021a) that included respectively 8, 12, and 69 studies demonstrated the prevalence of depression, anxiety, insomnia, or stress among health professionals. The reported prevalence estimates of psychological disorders during the COVID-19 pandemic are higher than the estimates of normal time (Pan et al., 2020; Xiong et al., 2020). The history of a pandemic that causes an enormous negative impact on physical and mental health and economies is very old (Qiu et al. 2017; Goulia et al., 2010). It may also have an association with higher psychological disorders in the current pandemic. During this pandemic, plenty of cross-sectional studies are emerging on the prevalence of psychological morbidity. The investigation on patterns of mental health rather than cross-sectional prevalence rates is more helpful to understand the psychological dysfunction and resilience (Chen \& Bonanno, 2020). There is also evidence that those psychological crises are changing periodically during the pandemic (Mahmud et al., 2021a). However, there is an absolute shortage of literature that identifies the periodic variation in psychological conditions. This has motivated the authors to investigate psychological outcomes among the general population over time during the pandemic. Here, the general population refers to non-healthcare, nonfirst responders who have not been infected with the coronavirus. The purpose of this systematic review is to analyze the existing research findings which are related to psychological issues depression, anxiety, stress, and insomnia during this COVID-19 pandemic among the general people. The study also investigates the periodic changes and region-wise variations in mental health conditions during the COVID-19 pandemic.

\section{Methods}

We have strictly followed Preferred Reporting Items for Systematic Reviews and Meta-Analysis (PRISMA) statements (Liberati et al., 2009) for conducting this systematic review. However, the review protocol was not previously registered. We have also followed the checklist of the Meta-analysis Of Observational Studies in Epidemiology (MOOSE) (Pappa et al., 2020).

\section{Search Strategy and Selection Criteria}

In this study, we created an Endnote (version X.8) library to catalog articles and remove duplicates. We have conducted a comprehensive systematic review using a systematic methodology (Fig. 1) for depression, anxiety, stress, as well as insomnia separately through the searches of PubMed, MEDLINE, and Google Scholar. The keywords that have been used in the systematic searches were "Coronavirus," "COVID-19," "2019-ncov," "SARS-cov-2," "Mental illness," "Mental health 


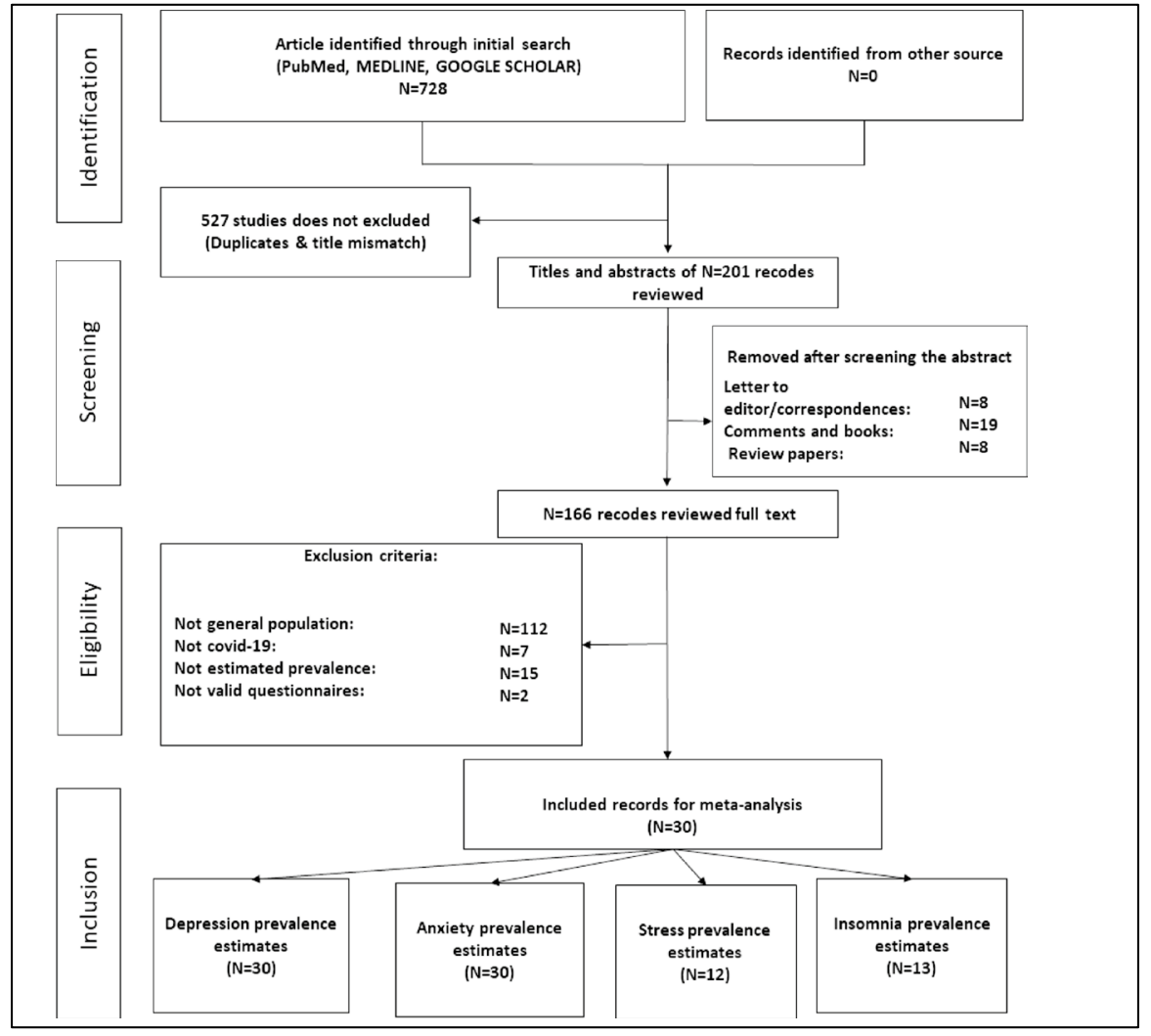

Fig. 1 Flowchart showing the stages of including/excluding study in the systematic review (Liberati et al., 2009) (PRISMA 2009)

problem," "Insomnia," "Distress," "Anxiety," "Depression," and "General population." All possible combinations of keywords have been used for searching the relevant articles by limiting the search to studies published after December 30, 2019, to before August 30, 2020. We also imposed the language barrier while selecting articles. The studies that were included in the analysis were published only in English. We did crosscheck the reference list of the selected articles to identify additional articles that met inclusion criteria. Moreover, the preprint papers published on Medrxiv, PsyArXiv, bioRxiv, and SSRN servers were also included.

\section{Inclusion/Exclusion Criteria}

The studies were included if and only if the study population or part of the study population is the general population. The studies were excluded from the database if they did not use validated measures or did not report study duration, study site, as well as sample size. The papers were excluded from the catalog in case of no English version, in case of no original data, and in case of no prevalence estimates 
of depression/anxiety/stress/insomnia were available. We also removed the reviews, letters to the editor, and correspondence.

\section{Quality Assessment}

Two independent authors (SM, and AM) evaluated the risk of bias of the included studies using a modified form of the Newcastle-Ottawa scale (Pappa et al., 2020), and a third author (ND) helped them resolve the potential disagreements. Pappa et al. (2020) modified the Newcastle-Ottawa scale by considering the representativeness of the sample, sample size, determination of depression, distress, anxiety, and insomnia, and the use of appropriate statistical tools. With the cutoff point 3, the quality assessment score of the modified Newcastle-Ottawa scale ranged between 0 and 5 . The quality assessment score of $\geq 3$ indicates lower publication bias. On the other hand, a study has a high publication bias if the corresponding quality assessment score is $<3$.

\section{Screening and Extraction}

The data were extracted by two independent authors (SM, and AM), with the presence of third reviews if necessary (MM). The first two authors screened all the articles (30) that satisfied the inclusion criteria and extracted data using a standardized form. The information extracted from the selected articles included article title, first author's name, year of publication, place of study, name of the authors, sampling method, duration of data collections, sample size, percentage of male respondents, assessment methods, the prevalence of depression, stress, anxiety, and insomnia.

\section{Outcomes and Measures}

Depression, anxiety, stress, and insomnia are the main outcomes of this systematic review. Clinical interviews or self-rated screening instruments/questionnaires have been used to diagnose these psychological outcomes. Most of the people were diagnosed using self-rated electronic questionnaires along with Beck Anxiety Inventory (BAI) (Magán et al., 2008); Beck Depression Inventory-II (BDI-II) (Beck et al., 1996); Acute Stress Disorder Scale (ASDS) (Bryant et al., 2000); Athens Insomnia Scale (AIS) (Soldatos et al., 2003); Depression, Anxiety, and Stress Scale (DASS21) (Akin \& Çetın, 2007); Center for Epidemiological Studies Depression (CES-D) (Hann et al., 1999; Radloff, 1977); Six-item K6 Screening (K-6-S) (Andersen et al., 2011); Perceived Stress Scale (PSS) (Lee 2012); Beck Anxiety Inventory (BAI) (Fydrich et al., 1992); Zung Self-Rating Depression Scale (SDS) (Biggs et al., 1978; Zung, 1965); Zung Self-Rating Anxiety Scale (SAS) (Zung, 1965); General Anxiety Disorder 7-item scale (GAD-7) (Spitzer et al., 2006); General Anxiety Disorder 2-item scale (GAD-2) (Wells, 2005); Patient Health Questionnaire depression module-9 (PHQ-9) (Derogatis \& Cleary, 1977); Patient Health Questionnaire depression module-2 (PHQ-2) (Kroenke et al., 2003); Pittsburgh Sleep Quality Index (PSQI) (Buysse et al., 1989); and Insomnia Severity Index (ISI) (Bastien et al., 2001). 


\section{Statistical Analysis}

The meta-analysis of the prevalence of depression, anxiety, stress, and insomnia among the general population was carried out by STATA, statistical software version 16. The significance of the hypothesis was tested using the $\mathrm{z}$ statistic (level of significance $p<0.05)$. The heterogeneity tests were considered with a $5 \%$ level of significance to measure the homogeneity of studies. Due to significant heterogeneity, the random-effects model was used to estimate the pooled prevalence of depression, anxiety, stress, as well as insomnia with $95 \%$ confidence intervals and the relative weight for each study. All the results of the meta-analysis were displayed in forest plots. The potential publication bias was inspected by using the funnel plot/ Egger's test (Egger et al., 1997). We also conducted the subgroup analysis based on study time/duration, study location country/region, and assessment methods to observe the prevalence of depression, anxiety, stress, and insomnia from different stratifications and inspect the source of heterogeneity. All the studies were classified into three groups based on study duration for conducting the subgroup analysis: Before February 2020, from March to April 2020, and from May to June 2020. The studies were also classified into different groups based on territories by following the WHO's regional classifications (Mahmud et al., 2021; WHO, n.d.).

\section{Results}

\section{Study Characteristics}

After the complete systematic selection procedure (depicted in Fig. 1) 30 studies (Ueda et al., 2020; Liu et al. 2020; Zhou et al. 2020a, 2020b, ; Sigdel et al. 2020; Kazmi et al., 2020; Othman, 2020; Shevlin et al., 2020; Odriozola-González et al., 2020; Agberotimi et al. 2020; Mazza et al., 2020; Shi et al., 2020; Rossi et al. 2020; Dai et al., 2020; Fu et al., 2020; Gualano et al., 2020; Tang et al. 2020; Huang \& Zhao, 2020; Marelli et al., 2020; McCracken et al., 2020; Song et al., 2020; Wang et al., 2020a, 2020b, 2020c, 2020d; Islam et al. 2020; Salman et al. 2020; Verma \& Mishra, 2020; Grover et al., 2020; Ozamiz-Etxebarria et al., 2020; Pieh, et al., 2020) with a total of 162,027 respondents were comprised in the analysis. Among all the respondents, on average, $41.14 \%$ were males. All the studies were cross-sectional and reported on anxiety, depression, stress, or insomnia. Most of the studies (70\%) used an online survey and $13 \%$ of the studies used web-based/social media sampling. The remaining $17 \%$ of studies either used convenience sampling or snowball sampling or respondent driven sampling (RDS) or random sampling. Out of 30 studies, 11 studies took place in China, 4 in Italy, 3 in India, 2 in Spain, and 1 study was undertaken in each of the countries namely Austria, Japan, Bangladesh, Iran, Malaysia, Nepal, Nigeria, Pakistan, Sweden, United Kingdom (UK). There were 7 preprint $(23.33 \%)$ and 23 published $(76.67 \%)$ papers included in this study and $43 \%$ (13/30) of similar studies were found in another systematic review (Salari et al., 2020). A total of 7 studies used DASS-21 tools for assessing depression, anxiety, and stress. PHQ-9 tools were considered in 18 studies and CES-D was considered 
in 3 studies for screening depression. 3 studies used three different assessment tools (SDS, PQH-2, BDI-II). For assessing anxiety, 19 studies used GAD-7, 4 studies used four different tools (SAS, CES-D, GAD-2, BAI). For assessing stress, 3 studies used PSS and another two studies used ASDS and K6-S. Nine studies considered ISI, two studies used AIS, and another two studies used PSQI for measuring the severity of insomnia. Brief characteristics for each study are provided in Table 1 which includes the sample size, study location, duration of the study, male/female ratio, sampling method, assessment method, the prevalence of depression, anxiety, stress, and insomnia. The Modified Newcastle-Ottawa quality (Pappa et al., 2020) assessment results show that the score for most of the studies (27) is greater than 3 which indicates there is lower or no publication bias for the corresponding study (Table 2). And the remaining 3 studies scored exactly 3 which also indicates a lower publication bias.

\section{Statistical Heterogeneity and Publication Bias}

Heterogeneity of the studies was investigated using $Q$-test and $I^{2}(\%)$ indices. We have found significant heterogeneity in our meta-analysis of effect of COVID-19 on depression $\left(Q=x_{(29)}^{2}=14,826.12, p<0.05\right)\left(I^{2}=99.75 \%, p<0.0001\right)$, anxiety $(Q=$ $\left.x^{2}(29)=10,806.67, p<0.05\right)\left(I^{2}=99.79 \%, p<0.0001\right)$, stress $\left(Q=x^{2}(11)=3612.96\right.$, $p<0.05)\left(I^{2}=99.89 \%, p<0.0001\right)$, and insomnia $\left(Q=x^{2}(12)=10,071.34, p<0.05\right)$ $\left(I^{2}=99.89 \%, p<0.0001\right)$. To evaluate the publication bias of the selected studies, the Funnel plot and Eggers's test indices for depression $(z=0.33, p=0.73)$ (Fig. 2a), anxiety $(z=1.38, p=0.17)$ (Fig. 2b), stress $(z=-0.01, p=0.98)$ (Fig. 2c), and insomnia $(z=1.82, p=0.067$ ) (Fig. $2 \mathrm{~d}$ ), which indicates that there is no publication bias for any of the four clinical symptoms.

\section{Prevalence}

The prevalence of depression among the general population was estimated using 30 studies. The estimated pooled prevalence was $28.18 \%$ (95\% CI: 23.81-32.54) for depression, presented in Fig. 3a. Similarly, 30 studies were used to estimate the pooled prevalence of anxiety during the COVID-19 pandemic among the general population. A pooled prevalence of $29.57 \%$ (95\% CI: 24.67-34.47) was estimated for anxiety, presented in Fig. 3b. The prevalence of stress was calculated using 13 studies, where a pooled prevalence of $25.18 \%$ (95\% CI: 14.82-35.54) was appraised (Fig. 3c). In the case of estimation of the prevalence of insomnia, 12 studies were used and we obtained a pooled prevalence of $23.50 \%$ (95\% CI: 16.44-30.57) (Fig. 3d).

\section{Subgroup Analysis Based on Study Periods}

Subgroup analysis of the prevalence of depression, anxiety, stress, and insomnia was done based on the study period. For depression, the pooled prevalence of the study periods before February 2020, March to April 2020, and May to June 2020 


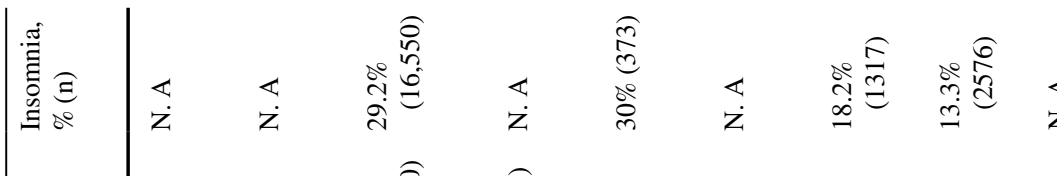

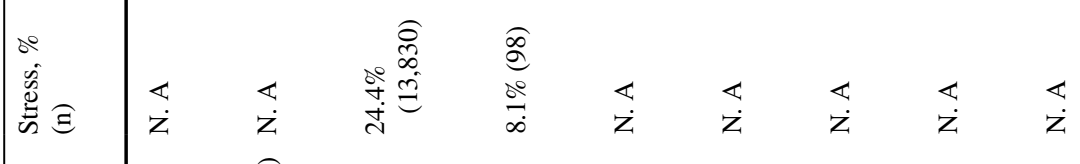

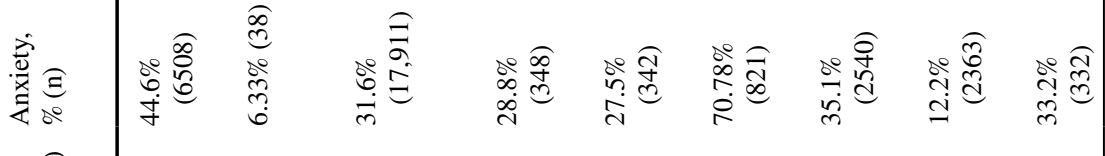

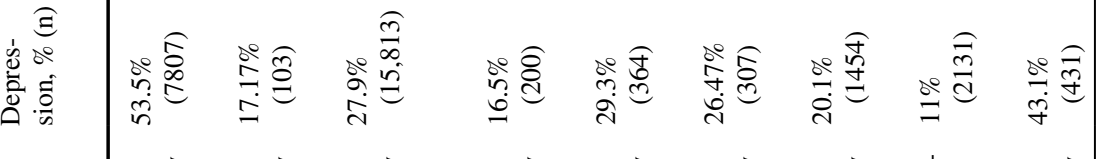

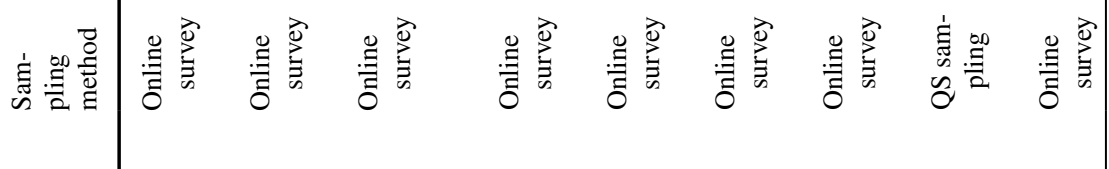

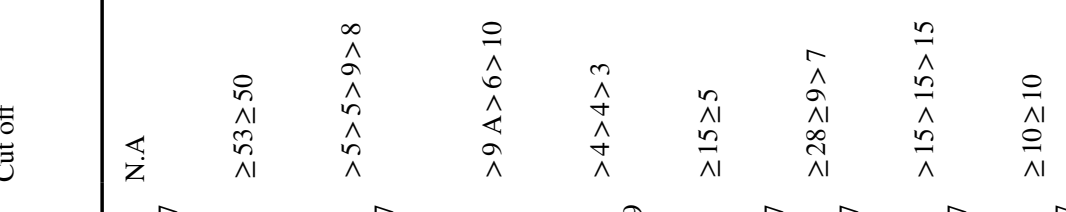

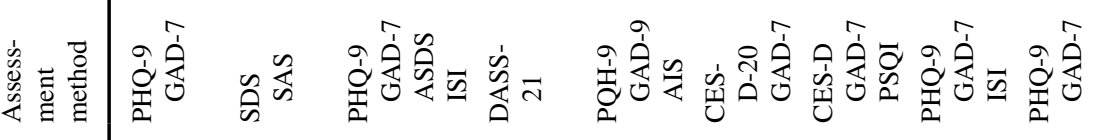

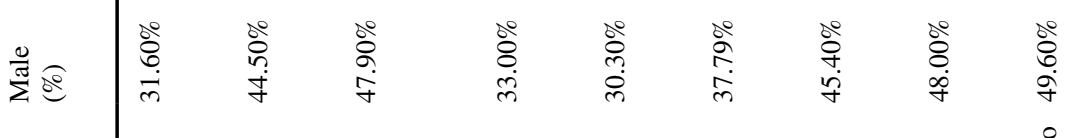

勇芯

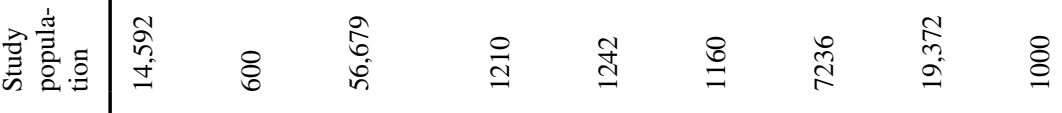

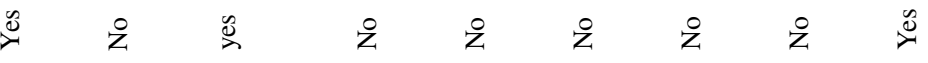

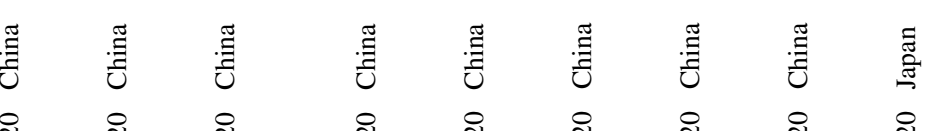

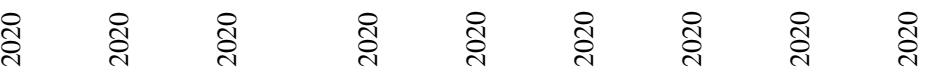

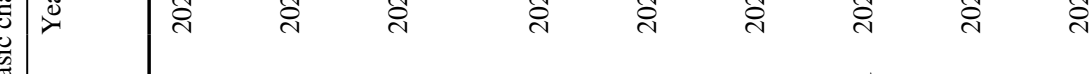

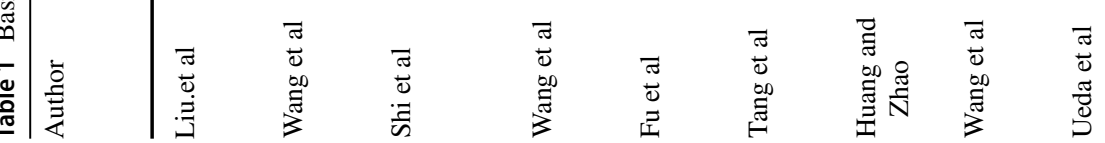




\begin{tabular}{|c|c|c|c|c|c|c|c|c|c|c|}
\hline 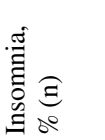 & $\begin{array}{l}\varangle \\
\dot{z}\end{array}$ & $\begin{array}{l}\varangle \\
z\end{array}$ & $\begin{array}{l}\varangle \\
z\end{array}$ & $\begin{array}{l}\varangle \\
Z\end{array}$ & $\begin{array}{l}\varangle \\
z\end{array}$ & $\begin{array}{l}\varangle \\
z\end{array}$ & $\begin{array}{l}0 \\
\triangleq \\
\therefore \\
\ddots\end{array}$ & $\begin{array}{l}\varangle \\
z\end{array}$ & 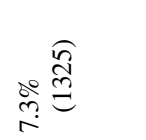 & 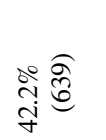 \\
\hline 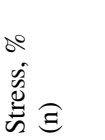 & & & $\begin{array}{l}\stackrel{a}{n} \\
\dot{n} \\
\dot{n}\end{array}$ & 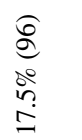 & $\begin{array}{l}\varangle \\
z\end{array}$ & $\underset{\infty}{\stackrel{\overbrace{}}{\stackrel{m}{m}}}$ & $\begin{array}{l}\varangle \\
z\end{array}$ & 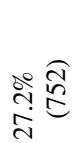 & 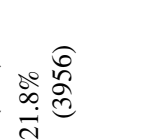 & $\begin{array}{l}\varangle \\
Z\end{array}$ \\
\hline 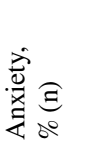 & 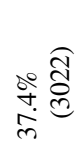 & $\begin{array}{l}\stackrel{0}{0} \\
\frac{0}{9}\end{array}$ & 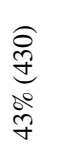 & 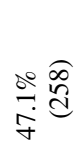 & 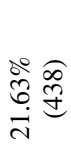 & 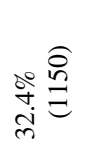 & $\begin{array}{l}\stackrel{0}{0} \underset{\mathfrak{q}}{d} \\
\stackrel{q}{d}\end{array}$ & $\begin{array}{l}\stackrel{E}{\sigma} \\
\stackrel{5}{\infty}\end{array}$ & 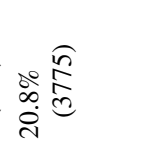 & $\begin{array}{l}\stackrel{\bar{n}}{2} \\
\text { ते }\end{array}$ \\
\hline 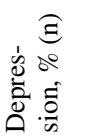 & 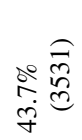 & $\begin{array}{l}\underset{\partial}{\Xi} \\
\text { } \\
\frac{0}{d}\end{array}$ & $\begin{array}{l}\stackrel{a}{a} \\
\hat{\infty} \\
\infty \\
\infty\end{array}$ & $\begin{array}{l}\stackrel{\sigma}{o} \\
\stackrel{d}{d}\end{array}$ & $\begin{array}{l}\stackrel{\circ}{\mathrm{N}} \\
\stackrel{\text { ते }}{+}\end{array}$ & 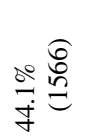 & 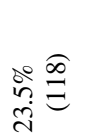 & $\begin{array}{l}\infty \widehat{\hat{\sigma}} \\
\infty \\
\text { ले }\end{array}$ & 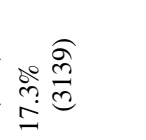 & 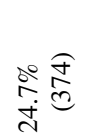 \\
\hline 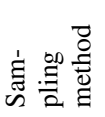 & 悬 & 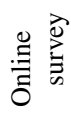 & 駡离 & 哥 离 & 弯 & 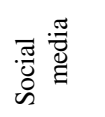 & $\begin{array}{l}\infty \\
\infty \\
\infty \\
\tilde{\alpha}\end{array}$ & 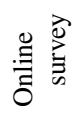 & : & 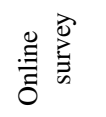 \\
\hline 芦 & $\stackrel{\stackrel{\Delta}{\wedge}}{\stackrel{\Delta}{\wedge}}$ & 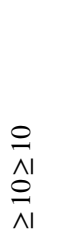 & $\begin{array}{l}\stackrel{0}{\wedge} \\
\hat{\wedge} \\
\hat{\Lambda} \\
\wedge\end{array}$ & $\begin{array}{l}\stackrel{0}{\wedge} \\
\hat{\wedge} \\
\hat{\Lambda} \\
\hat{\wedge}\end{array}$ & $\begin{array}{l}\stackrel{0}{1} \\
\hat{\wedge} \\
\stackrel{1}{\Lambda} \\
\wedge 1\end{array}$ & $\begin{array}{l}\stackrel{0}{\wedge} \\
\hat{b} \\
\hat{\Lambda} \\
\hat{\Lambda}\end{array}$ & $\begin{array}{l}\hat{\lambda} \\
\hat{O} \\
\hat{\wedge} \\
\hat{n}\end{array}$ & $\begin{array}{l}\circ \\
\hat{\Lambda} \\
\hat{0} \\
\hat{\Lambda} \\
\wedge\end{array}$ & $\begin{array}{l}\hat{N} \\
\hat{\imath} \\
\hat{\theta} \\
\hat{n} \\
\hat{n} \\
\hat{n} \\
\hat{\wedge}\end{array}$ & $\begin{array}{l}\stackrel{+}{\wedge} \\
\stackrel{\wedge}{N} \\
\wedge\end{array}$ \\
\hline 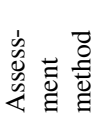 & 完余 & 离交 & $\frac{\dot{s}}{\hat{n}} \bar{c}$ & 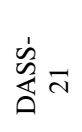 & 产空 & $\frac{\dot{v}}{\hat{\omega}} \vec{\Delta}$ & 产㹸氙 & $\frac{\dot{s}}{\dot{n}} \vec{\imath}$ & 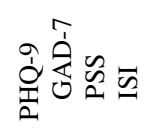 & 紊灾氛 \\
\hline$\sum_{\bar{\pi}}^{\frac{\pi}{\pi}} \widehat{\varrho}$ & 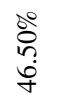 & $\begin{array}{l}\stackrel{8}{0} \\
\text { ì } \\
\text { in }\end{array}$ & $\begin{array}{l}\stackrel{\circ}{0} \\
\stackrel{0}{\infty} \\
\infty\end{array}$ & $\begin{array}{l}\stackrel{0}{0} \\
\dot{0} \\
\dot{\sigma}\end{array}$ & $\begin{array}{l}\stackrel{0}{8} \\
8 \\
\dot{\infty}\end{array}$ & $\begin{array}{l}\stackrel{\circ}{0} \\
\dot{m} \\
m\end{array}$ & $\begin{array}{l}80 \\
\text { bे } \\
\text { ñ }\end{array}$ & $\begin{array}{l}\stackrel{0}{0} \\
\text { oे } \\
\infty \\
\text { i }\end{array}$ & $\begin{array}{l}\stackrel{0}{0} \\
\stackrel{+}{\dot{\alpha}} \\
\text { in }\end{array}$ & $\begin{array}{l}\stackrel{\circ}{0} \\
\stackrel{+}{+} \\
\dot{m}\end{array}$ \\
\hline 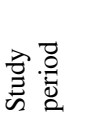 & 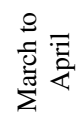 & 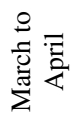 & 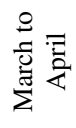 & 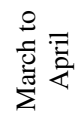 & 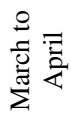 & 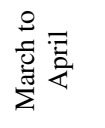 & 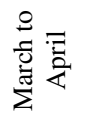 & 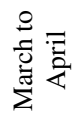 & 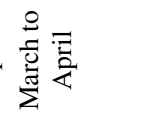 & 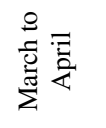 \\
\hline 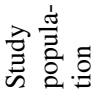 & $\stackrel{\hat{\sigma}}{\infty}$ & 守 & $\S$ & $\stackrel{\infty}{i}$ & 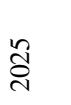 & $\begin{array}{l}\stackrel{n}{n} \\
n \\
m\end{array}$ & ชి & 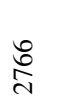 & $\begin{array}{l}\hat{ક} \\
\infty \\
\infty\end{array}$ & $\frac{n}{n}$ \\
\hline 号 & $\stackrel{2}{z}$ & $\stackrel{0}{\approx}$ & 之 & $\stackrel{\circ}{z}$ & $\stackrel{\circ}{z}$ & $\stackrel{0}{0}$ & $\stackrel{\triangleq}{\circlearrowright}$ & z & $\stackrel{2}{z}$ & $\dot{z}$ \\
\hline : ఫ్ర్లే & 节 & $\begin{array}{l}\overline{\tilde{Q}} \\
\text { 己े }\end{array}$ & $\stackrel{g}{ت}$ & $\underset{\mathbb{G}}{\mathscr{G}}$ & 光 & $\begin{array}{l}\text { 苛 } \\
\text { की }\end{array}$ & 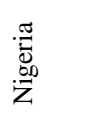 & 吾 & 吾 & 胥 \\
\hline こ्र & ठัญ & 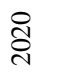 & ્ֻતి & 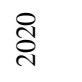 & ֻัญి & ્ֻતે & ๙ัญ & ๕ัญ & ๙ัరి & ๙ัญ \\
\hline $\begin{array}{l}\grave{\vdots} \\
\Xi\end{array}$ & 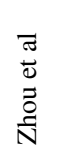 & 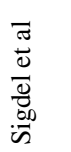 & 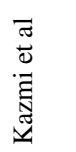 & 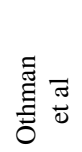 & 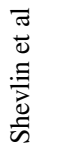 & 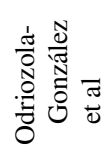 & 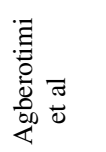 & 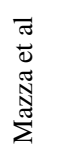 & 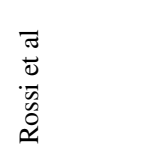 & 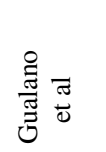 \\
\hline
\end{tabular}




\begin{tabular}{|c|c|c|c|c|c|c|c|c|}
\hline 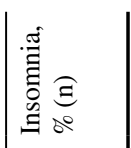 & $\begin{array}{l}\hat{a} \\
\stackrel{0}{a} \\
\stackrel{0}{a}\end{array}$ & 总 & $\begin{array}{l}\widehat{0} \\
i \stackrel{0}{0} \\
\dot{j}\end{array}$ & $\begin{array}{l}\overleftrightarrow{Z} \\
z\end{array}$ & $\begin{array}{l}\varangle \\
z\end{array}$ & $\begin{array}{l}\overleftrightarrow{Z} \\
\dot{z}\end{array}$ & $\underset{\overparen{\sigma}}{\stackrel{\sigma}{0}}$ & 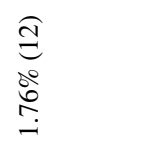 \\
\hline 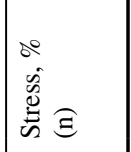 & $\begin{array}{l}\overleftrightarrow{Z} \\
\dot{z}\end{array}$ & 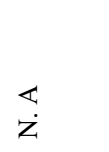 & $\begin{array}{l}\varangle \\
\dot{z}\end{array}$ & $\begin{array}{l}\widehat{\vartheta} \\
\stackrel{g}{\circ} \\
\stackrel{0}{=}\end{array}$ & 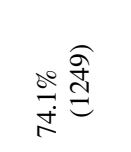 & 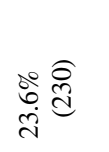 & $\begin{array}{l}\underset{\sigma}{\sigma} \\
\underset{0}{0}\end{array}$ & 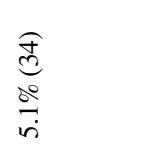 \\
\hline 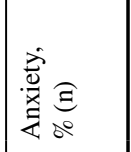 & 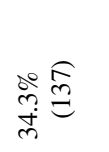 & 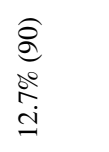 & $\underset{\substack{\dot{j} \\
\dot{j}}}{\widehat{F}}$ & 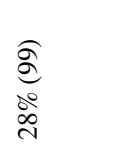 & $\begin{array}{l}\stackrel{0}{q} \\
\stackrel{d}{d}\end{array}$ & 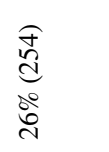 & $\begin{array}{l}\widehat{a} \\
\stackrel{a}{a}\end{array}$ & 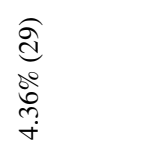 \\
\hline 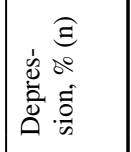 & $\begin{array}{l}\infty \\
\stackrel{\sim}{\sim} \\
\stackrel{\sim}{\Xi}\end{array}$ & $\begin{array}{l}\stackrel{0}{0} \\
\stackrel{0}{0} \\
\stackrel{2}{2}\end{array}$ & $\begin{array}{l}\stackrel{f}{\vec{n}} \\
\dot{f}\end{array}$ & 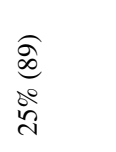 & $\begin{array}{l}\text { } \\
\text { 这 } \\
\stackrel{\circ}{\circ}\end{array}$ & $\begin{array}{l}\stackrel{f}{\Phi} \\
\stackrel{\infty}{=}\end{array}$ & $\begin{array}{l}\widehat{\vec{a}} \\
\stackrel{\vec{d}}{a} \\
\stackrel{\vec{d}}{ }\end{array}$ & 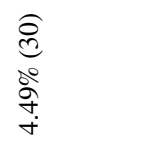 \\
\hline 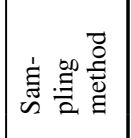 & 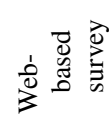 & 密密量 & 急 & 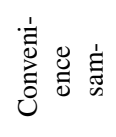 & 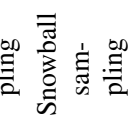 & 急 & 号 & 号害 \\
\hline 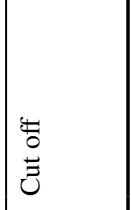 & $\begin{array}{l}\hat{n} \\
\hat{\Lambda} \\
\hat{n} \\
\hat{\Lambda}\end{array}$ & $\begin{array}{l}\infty \\
\hat{\Lambda} \\
\hat{\Lambda} \\
\Delta \\
\wedge\end{array}$ & $\begin{array}{l}n \\
\hat{n} \\
\hat{n}\end{array}$ & $\begin{array}{l} \pm \\
\hat{\lambda} \\
\hat{\lambda} \\
\hat{\Lambda}\end{array}$ & $\begin{array}{l}\stackrel{M}{\wedge} \\
\hat{A} \\
\hat{A} \\
\wedge\end{array}$ & 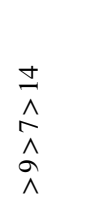 & $\begin{array}{l}\stackrel{n}{\wedge} \\
\hat{a} \\
\hat{a} \\
\stackrel{\wedge}{\wedge}\end{array}$ & $\begin{array}{l}\hat{n} \\
\hat{n} \\
\hat{n} \\
\hat{n}\end{array}$ \\
\hline 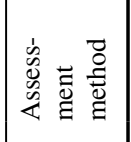 & 窝离氙 & 窐完氙 & 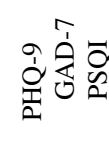 & $\hat{⿱ 亠 凶}_{\vec{\Delta}} \vec{N}$ & 家仝怘 & पे & 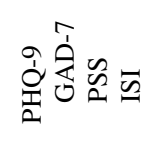 & 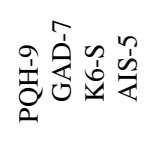 \\
\hline$\frac{0}{\pi_{\pi}^{\pi}}$ & 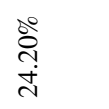 & $\begin{array}{l}\text { @े } \\
\infty \\
\text { ¿ें }\end{array}$ & $\frac{\stackrel{9}{9}}{\stackrel{9}{a}}$ & $\frac{\stackrel{0}{\stackrel{2}{i n}}}{i}$ & $\begin{array}{l}\stackrel{8}{R} \\
\grave{0}\end{array}$ & $\begin{array}{l}\stackrel{0}{\circ} \\
\infty \\
\infty\end{array}$ & $\begin{array}{l}\stackrel{8}{0} \\
\stackrel{+}{+}\end{array}$ & $\begin{array}{l}\dot{\infty} \\
\dot{\sigma} \\
\dot{q}\end{array}$ \\
\hline 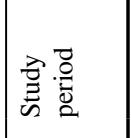 & 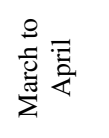 & 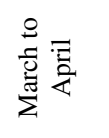 & 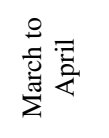 & 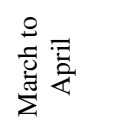 & 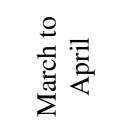 & 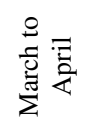 & 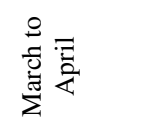 & 疍息 \\
\hline 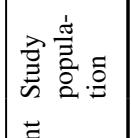 & \& & $\stackrel{?}{尺}$ & $\begin{array}{l}\mathscr{\infty} \\
\infty \\
=\end{array}$ & 芦 & $\stackrel{\mathscr{0}}{\mathscr{0}}$ & 怘 & $\stackrel{\approx}{\varrho}$ & : \\
\hline D. & $\stackrel{\circ}{z}$ & $\stackrel{2}{z}$ & $\stackrel{2}{z}$ & $\stackrel{2}{z}$ & z̊ & $\stackrel{\circ}{z}$ & z̊ & $\stackrel{2}{z}$ \\
\hline . & 产 & हี & 苞 & : & $\stackrel{\pi}{\overparen{\pi}}$ & $\begin{array}{l}\text { : } \\
\text { कू }\end{array}$ & $\frac{\tilde{g}}{\bar{z}}$ & 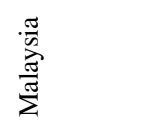 \\
\hline$z$ & స్తి & ڤ్తి & ฮิ & ণ్ָి & ๙ิેి & ฮิ & ฮิ & ฮ్రి \\
\hline 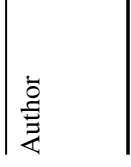 & 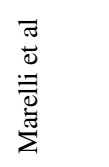 & $\begin{array}{l}\bar{\sigma} \\
\tilde{0} \\
\infty \\
\overline{0} \\
\omega\end{array}$ & $\begin{array}{l}\bar{\Xi} \\
\overline{0} \\
\overline{0} \\
\frac{0}{N}\end{array}$ & 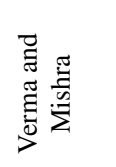 & 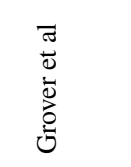 & 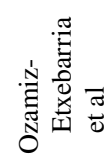 & $\begin{array}{l}\pi \\
\frac{\pi}{0} \\
\frac{\pi}{0} \\
: 0\end{array}$ & $\begin{array}{l}\bar{\Xi} \\
\tilde{0} \\
\tilde{\tilde{D}}\end{array}$ \\
\hline
\end{tabular}




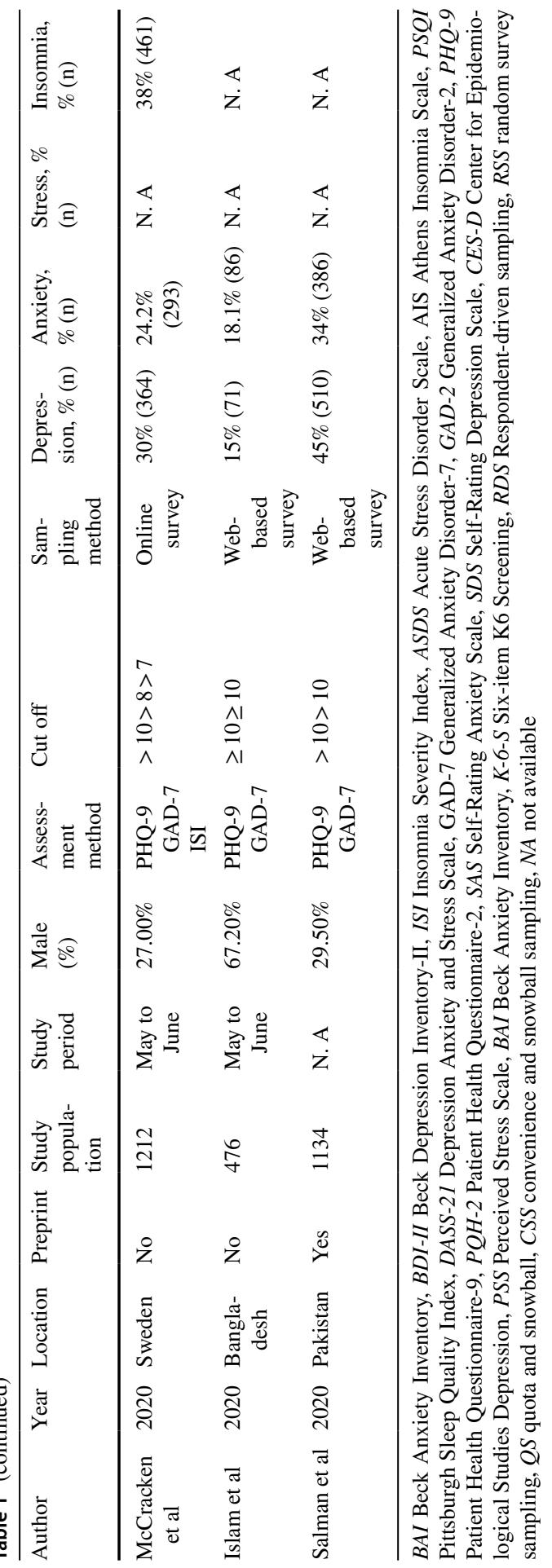




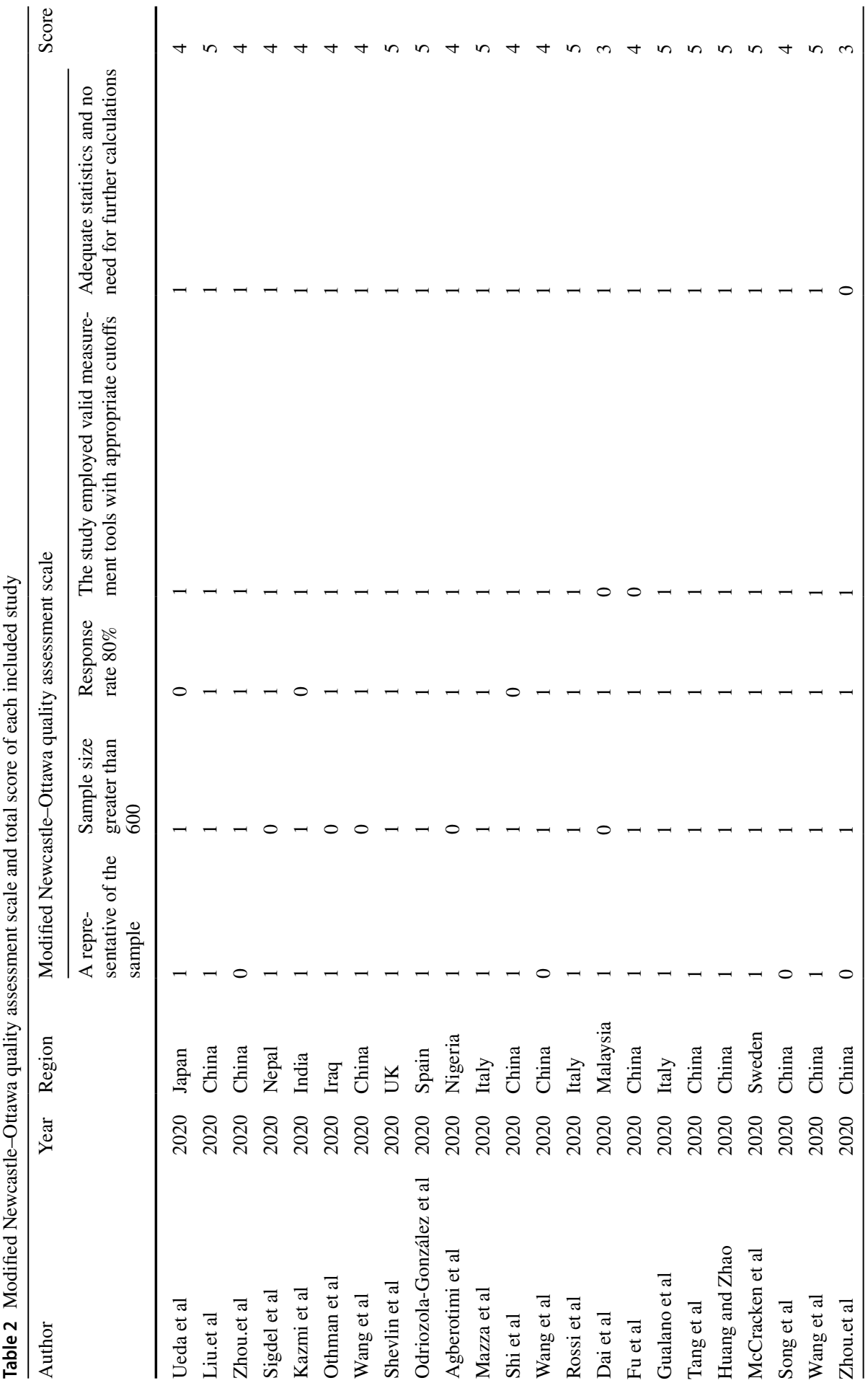




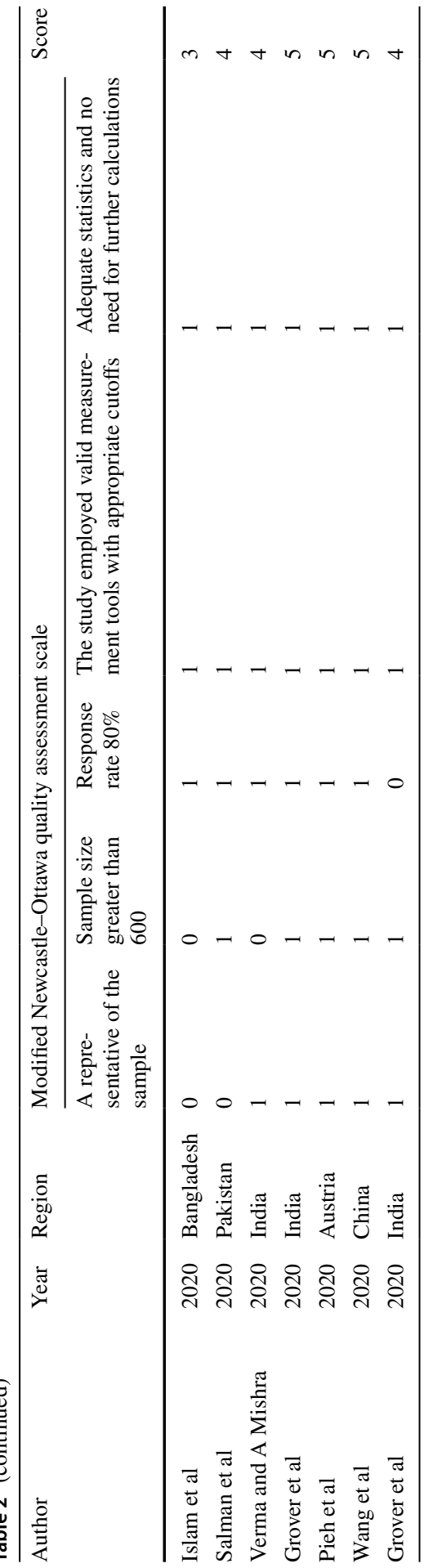




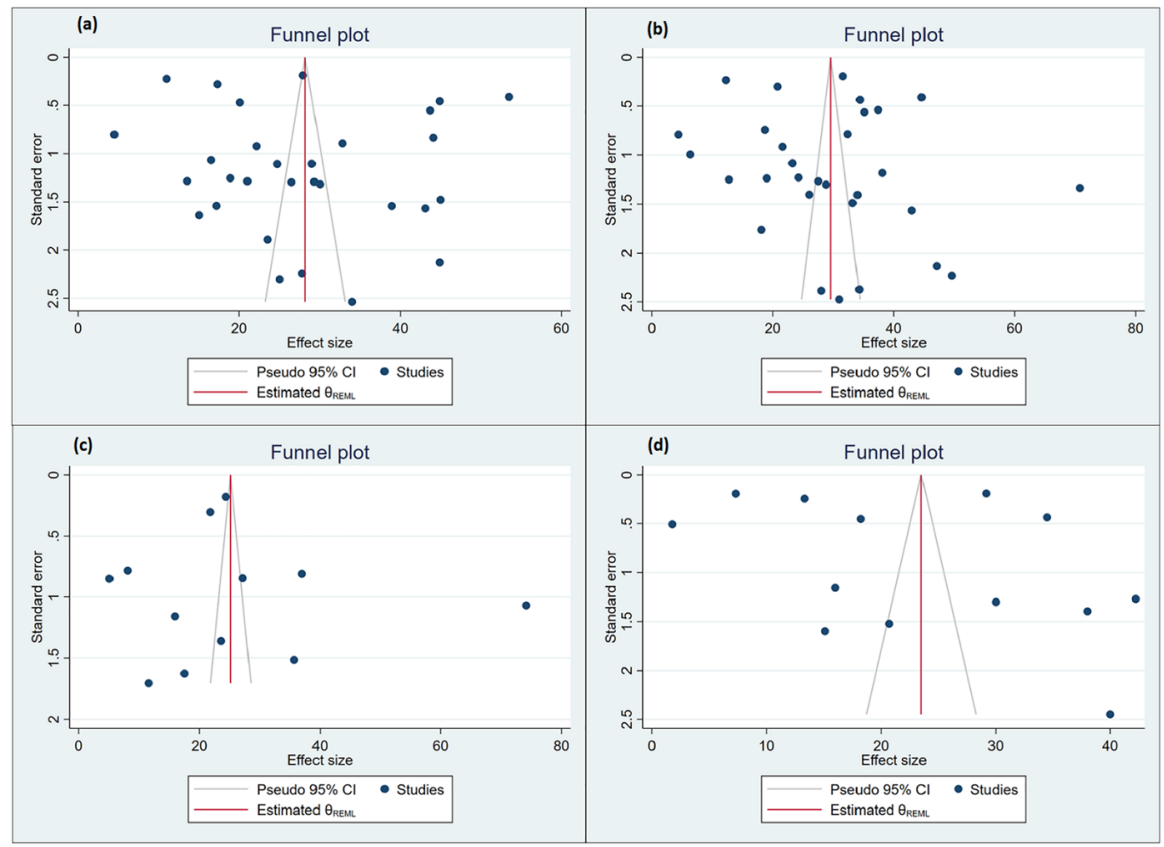

Fig. 2 Funnel plot of result of the prevalence of depression (a), anxiety (b), stress (c), and insomnia (d) among the general population

was $25.25 \%$ (95\% CI: 16.17-34.34), 30.51\% (95\% CI: 25.60-35.42), and $16.47 \%$ (95\% CI: 1.93-31.02), respectively (Fig. 4a). In the case of anxiety, the pooled prevalence of the study periods before February 2020, March to April 2020, and May to June 2020 were $32.10 \%$ (95\% CI: 18.37-45.83), 30.51\% (95\% CI: 25.79-35.23), and 15.51\% (95\% CI: 3.93-27.09), respectively (Fig. 4b). For stress, the pooled prevalence of the study periods before February 2020, March to April 2020, and May to June 2020 were $16.27 \%$ (95\% CI: 0.29-32.24), 29.41\% (95\% CI: 18.71-40.10), and $5.10 \%$ (95\% CI: 3.43-6.77), respectively (Fig. 4c). Similarly, in the case of insomnia, the pooled prevalence of the study periods before February 2020, March to April 2020, and May to June 2020 was $22.63 \%$ (95\% CI: 14.55-30.72), 25\% (95\% CI: 14.85-35.15), and 19.86\% (95\% CI: - 15.66-55.37), respectively (Fig. 4d).

According to our pooled prevalence estimates (Fig. 5) from the subgroup analysis based on time, the prevalence of depression among the general population reached its peak to 30.51\% during March and April 2020 from 25.25\% before February 2020 and then decreased by almost half (16.47\%) during May and June 2020. The prevalence of anxiety decreased substantially among the general population from $32.10 \%$ during December 2019 and February 2020 to 30.51\% during March and April 2020 and then to $15.51 \%$ during May and June 2020. In the case of stress, the prevalence increased during March and April 2020 (29.41\%) from the beginning of the pandemic (before February 2020, 16.27\%) and then decreased substantially during May and June $2020(5.10 \%)$. The prevalence estimate of insomnia also had a similar trend. It was highest during March and April 2020 (25\%) increasing from 22.63\% 


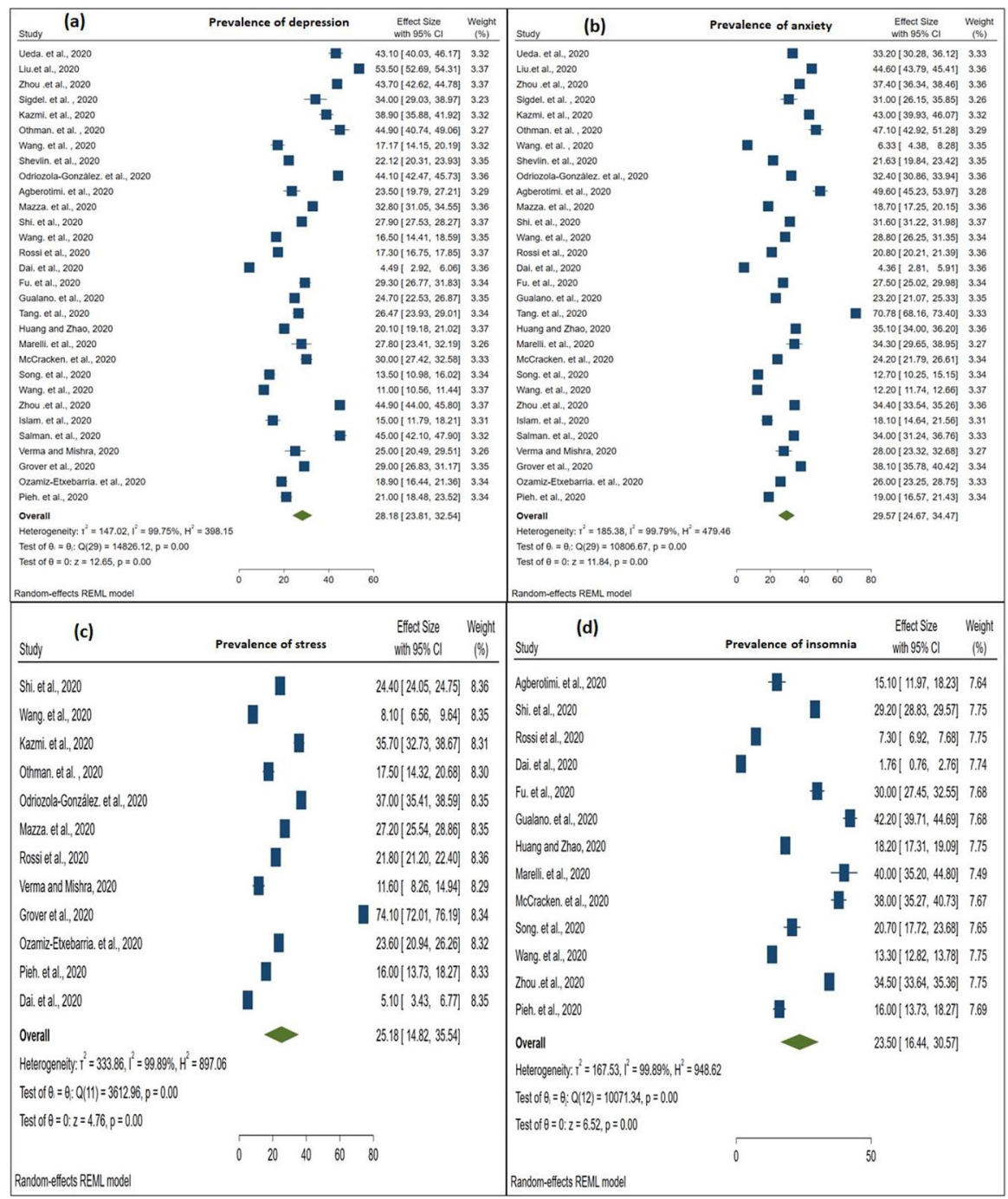

Fig. 3 Forest plot showing the meta-analyses of the pooled prevalence of depression (a), anxiety (b), stress (c), and insomnia (d) among the general population

before February 2020 and then decreased considerably to $19.86 \%$ during May and June 2020.

\section{Subgroup Analysis Based on Assessment Tools}

Another subgroup analysis of the prevalence of depression, anxiety, stress, and insomnia was done using assessment tools. For depression, pooled prevalence of assessment tools DASS-21, PHQ-9, and Others (SDS, PQH-2, BDI-II, CES-D) were 


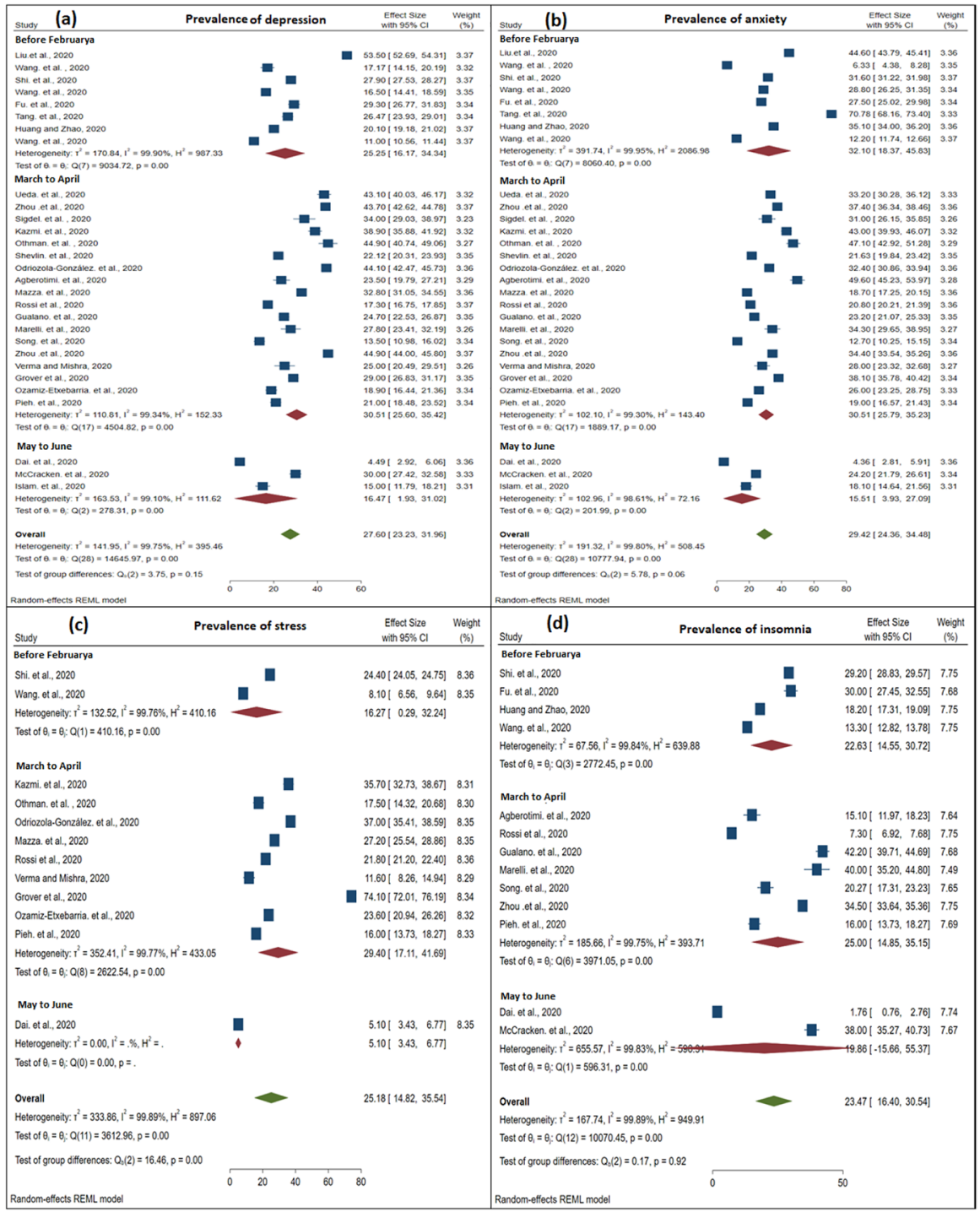

Fig. 4 Forest plot showing the meta-analyses of the pooled prevalence of depression (a), anxiety (b), stress (c), and insomnia (d) in different periods (December 2019 to June 2020)

respectively 31.57\% (95\% CI: 22.89-40.25), 29.10\% (95\% CI: 22.62-35.58), and $21.53 \%$ (95\% CI: 17.08-25.98) (Fig. 6a). In case of anxiety, pooled prevalence of assessment tools DASS-21, GAD-7, and Others (SDS, CES-D, GAD-2, BAI) was respectively $31.93 \%$ (95\% CI: 24.61-39.25), 30.13\% (95\% CI: 23.28-36.97), and $22.73 \%$ (95\% CI: 11.07-34.40) (Fig. 6b). For stress, pooled prevalence of assessment tools ASDS, DASS-21, K6-S, and PSS was 24.40\% (95\% CI: 24.05-24.75), $22.97 \%$ (95\% CI: $14.63-31.31$ ), 5.10\% (95\% CI: 3.43-6.77), and $37.30 \%$ (95\% CI: 


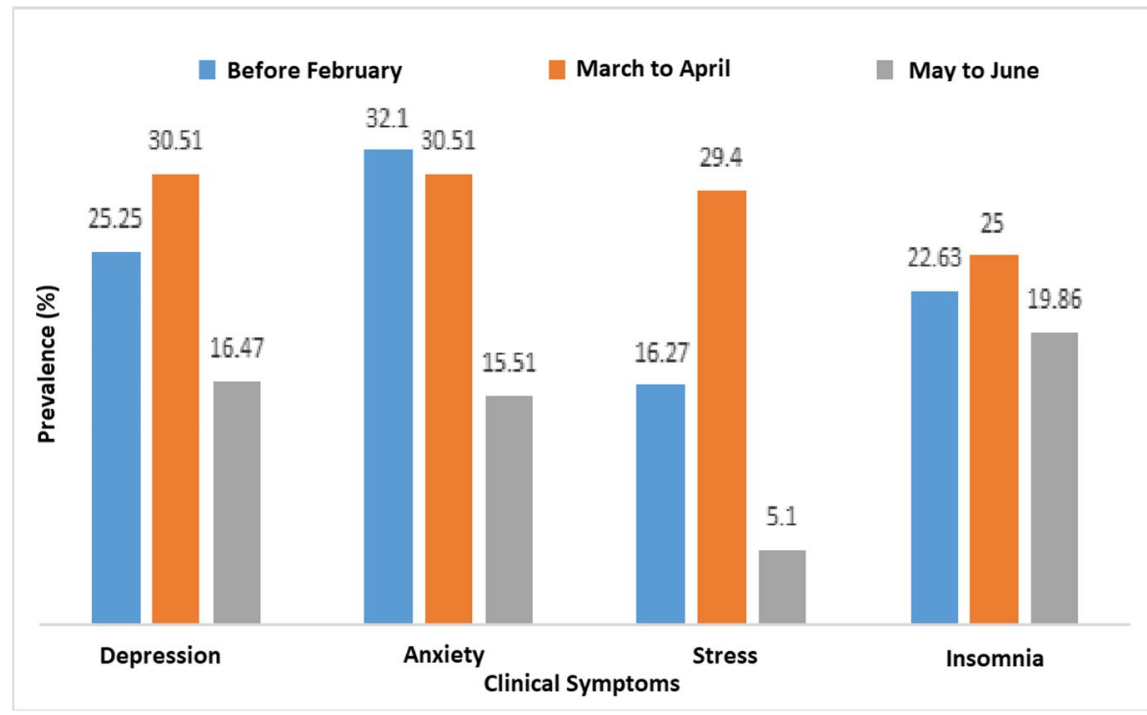

Fig. 5 Estimated prevalence of depression, anxiety, stress, and insomnia among general population during December 2019 to June 2020

1.09-73.50), respectively (Fig. 6c). Similarly, in case of insomnia, pooled prevalence of assessment tools AIS, ISI, and PSQL was 15.58\% (95\% CI: 0.76-2.76), $24.53 \%$ (95\% CI: 17.39-32.75), and 26.35\% (95\% CI: 10.38-42.32), respectively (Fig. 6d).

\section{Subgroup Analysis Based on Geographic Region and Countries}

To compare the findings among different countries and regions, we have defined different subgroups of the studies based on study locations (Six regions of WHO) namely: African Region, Eastern Mediterranean Region, European Region, Southeast Asia Region, and Western Pacific Region (no study was found from the Region of the Americas). Table 3 illustrates the regional and country-wise comparison of the prevalence of depression, anxiety, stress, and insomnia. The highest prevalence of depression, anxiety, stress, and insomnia was found respectively in Eastern Mediterranean Region (44.90\%, 95\% CI: 40.74-4906), African Region (49.60\%, 95\% CI: 45.23-53.97), Southeast Asia Region (40.49\%, 95\% CI: 4.49-76.16), and European Region $(31.18 \%$, 95\% CI: 15.57-48.04). On the other hand, Western Pacific Region (13.42\% 95\% CI: 4.90-21.94) and European Region (24.97\%, 95\% CI: 21.24-28.70) showed respectively the lowest prevalence of anxiety and stress. African Region showed the lowest prevalence of depression (15\% 95\% CI:11.97-18.23) and insomnia $(23.50 \%, 95 \%$ CI: 19.79-27.21). However, the between-country comparisons show that Malaysia has the lowest prevalence of depression $(4.49 \%$, 95\% CI: 2.92-6.06), anxiety (4.36\%, 95\% CI: 2.81-5.91), stress $(5.10 \%, 95 \%$ 


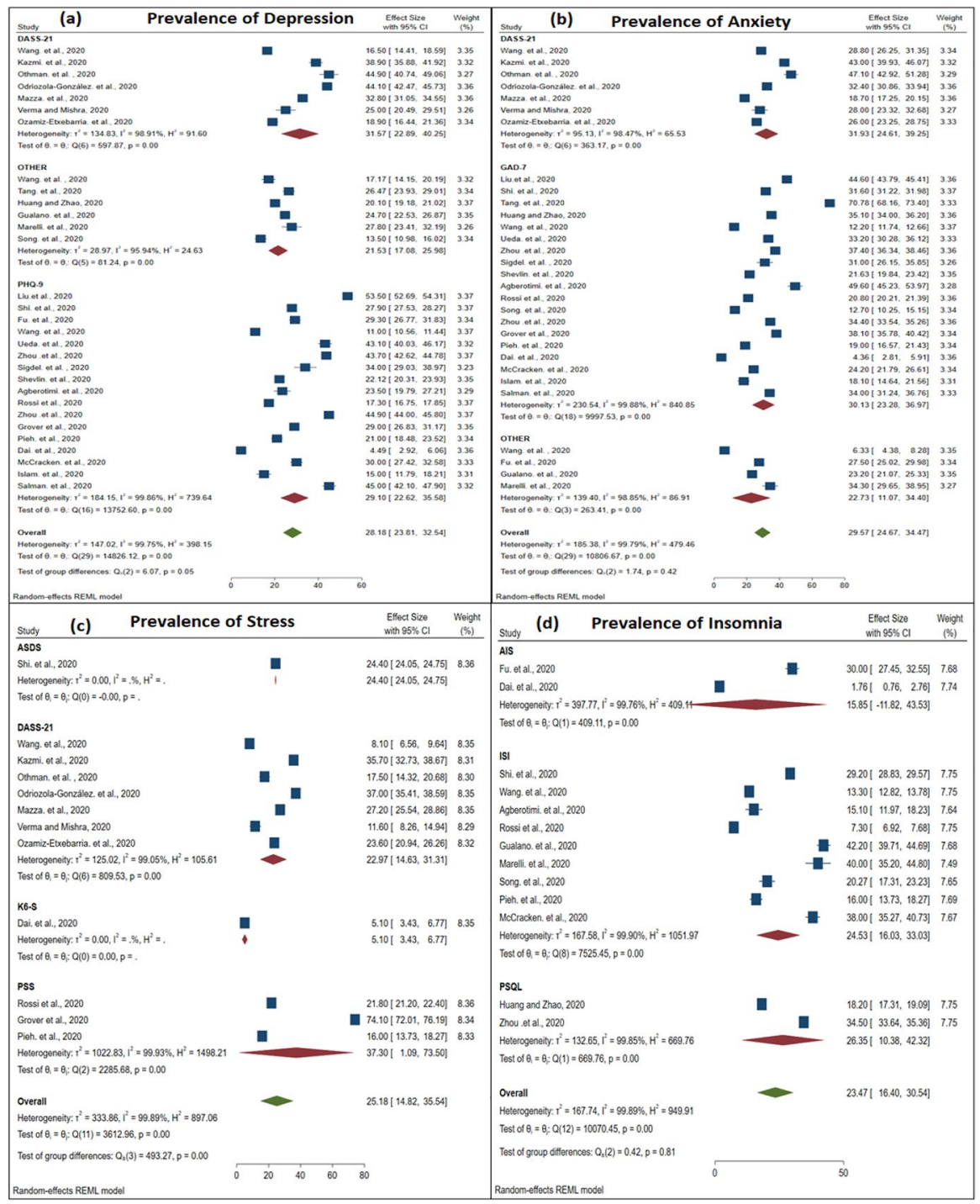

Fig. 6 Forest plot showing the meta-analyses of the pooled prevalence of depression (a), anxiety (b), stress (c), and insomnia (d) for different assessment methods

CI: 3.43-6.77), and insomnia (1.76\%, 95\% CI: 0.76-2.76). Studies in Pakistan reported the highest prevalence of depression (45\%, 95\% CI: 42.10-47.90) and anxiety (34\%, 95\% CI: 31.24-36.76). The highest prevalence of stress and insomnia were reported respectively in India $(40 \%, 95 \%$ CI: $4.81-76.16)$ and Italy $(29.76 \%, 95 \%$ CI: $7.57-51.96)$. 


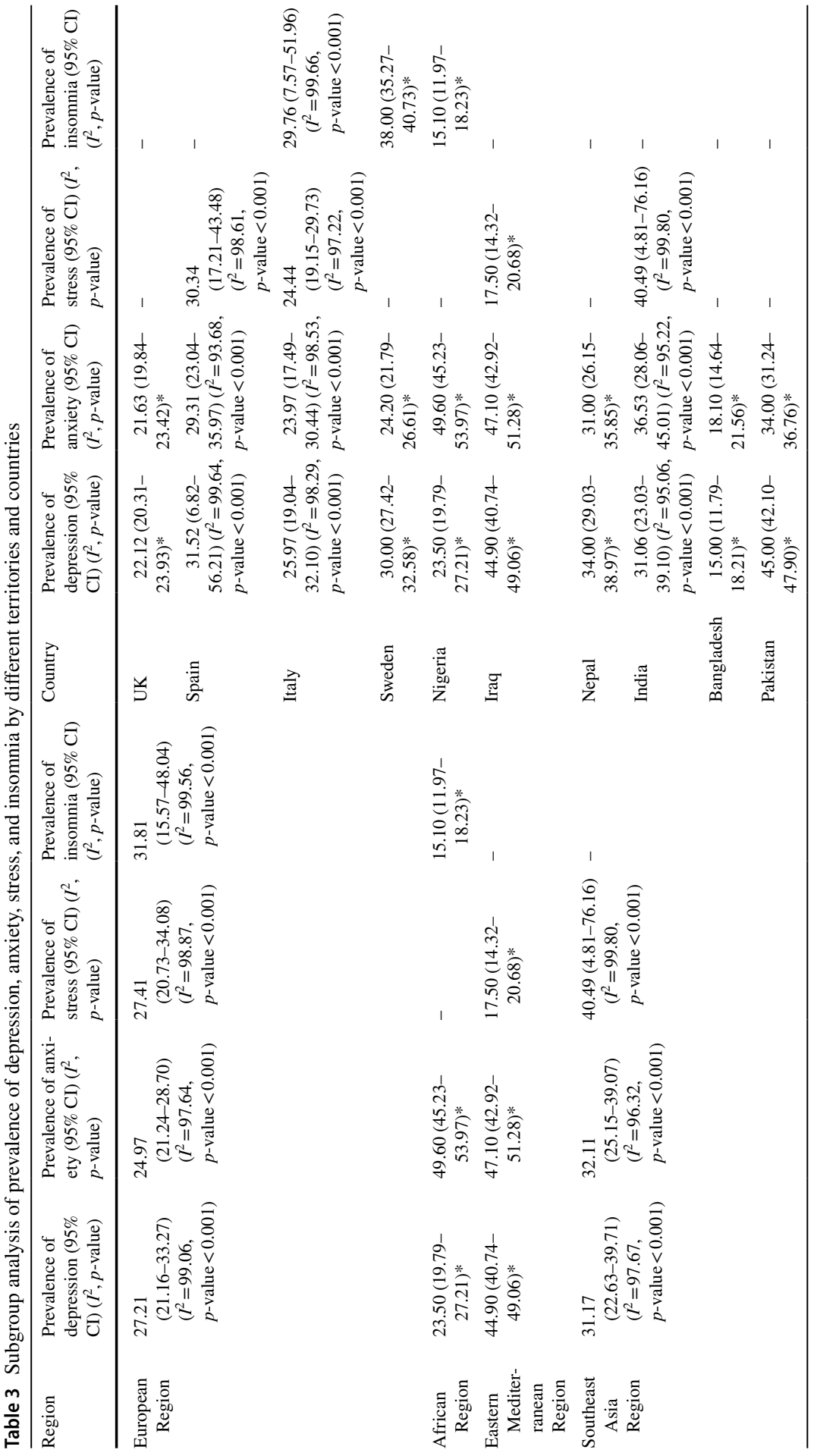




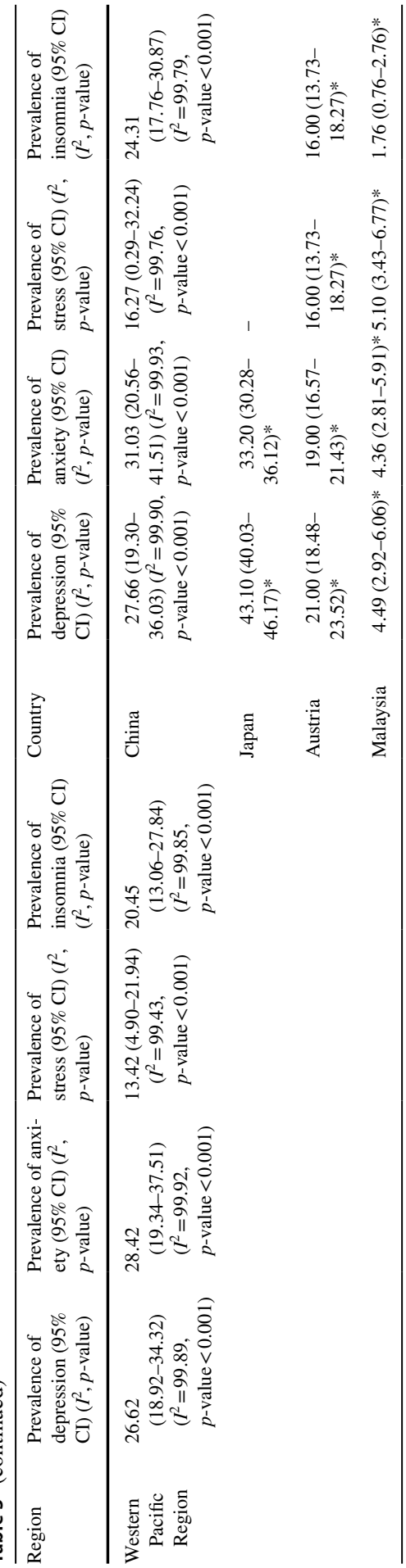




\section{Discussion}

This meta-analysis investigated the mental health difficulties of general people during the COVID-19 pandemic. It analyzed the prevalence of depression, anxiety, stress, and insomnia segregated by two periods and by WHO regions. This study followed the PRISMA and MOOSE checklists. All the studies included in the data analysis were cross-sectional. According to results from our data synthesis, during the COVID-19 pandemic, the prevalence of depression, anxiety, stress, and insomnia respectively was $28.18 \%, 29.57 \%, 20.18 \%$, and $23.50 \%$ in the general population. These psychiatric prevalence estimates are notably higher compared to before-pandemic situations (Huang et al., 2019; Krishnamoorthy et al., 2020; Lim et al., 2018). A previous meta-analysis found a similar prevalence of anxiety of $30 \%$ and a slightly higher prevalence of depression of 33\% among the general population (Wang, Di, et al., 2020; Wang, Kala, et al., 2020; Wang, Pan, et al., 2020). Comparatively, a higher prevalence of stress of $29.6 \%$ and an almost similar prevalence of depression and anxiety were found in another systematic review and meta-analysis (Salari et al., 2020). However, none of them showed the over-time changes and regional disparities among those psychological morbidities.

These intensified symptoms of COVID-19-related depression, anxiety, stress, and insomnia could be attributed to a result of psychosocial stressors such as life disturbance, disease concern, or fear of negative economic consequences. The prolonged quarantine/isolation time is also a potential explanation for such a high burden during the pandemic period (Krishnamoorthy et al., 2020). Among those who endure it, quarantine is an uncomfortable experience. Stress factors linked to finances, work, school closure, and stigma attached to the disorder may also be present. Previous studies of the psychological effects of quarantine during previous outbreaks showed that the incidence among the general population under quarantine was substantially higher (Brooks et al., 2020). Social media/news is also identified as a reason behind the higher prevalence of anxiety and stress during the pandemic (Gao et al., 2020). The perception of risk, mortality rate, food insecurity, stigma, and prejudice are major factors responsible for high psychological disorders among infected patients (Krishnamoorthy et al., 2020). Moreover, as there is no definite therapeutic agent or vaccine (as of the study) for COVID-19, there is still ambiguity about the outcome among patients that can further aggravate their mental status.

The subgroup analysis of the prevalence of depression, anxiety, stress, and insomnia based on the study period shows that prevalence is decreasing over time (see in Fig. 5). Our findings show that the prevalence of depression at the beginning of the COVID-19 pandemic, "Before February 2020," was 25.25\%; during "March to April 2020," it was $30.51 \%$ which is the highest prevalence followed by $16.47 \%$ in May to June 2020 (Fig. 4a). A similar pattern was found for stress and insomnia (Fig. 5). At the beginning of the pandemic, "Before February 2020," the prevalence of stress was $16.27 \%$ that rose to $29.41 \%$ during "March to April" followed by $5.10 \%$ in May to June 2020. The prevalence of insomnia before February 2020 was $22.63 \%$ that increased to 25\% in March and April 2020 and fell to 19.86\% in May and June 2020. However, in the case of anxiety, people were more anxious before February 2020 (32.10\%), slightly decreased in March and April 2020 (30.51\%), and 
then decreased to half (15.51\%) during May and June 2020. The pandemic spread all over the world after February 2020 and lockdowns in almost all countries and territories of the world started from the beginning of March 2020 (early lockdowns in China and some western nations). And after April 2020, people across the globe probably started to cope with the psychological challenges the pandemic poses. This might be the rationale behind the highest prevalence of depression, stress, and insomnia during March and April 2020 and an abatement thereafter. Several studies also reported a higher prevalence of psychological outcomes when individuals were challenged by isolation, unexpected unemployment, and economic uncertainty associated with the pandemic (Xiong et al., 2020). Before February 2020, people all over the world became more anxious by the news of the invention of a new case of atypical pneumonia (previous version of COVID-19) that was reported in Wuhan, China (Anand et al. 2020). And over time, it made people more depressed, stressful, and sleepless.

The subgroup analysis also provides the changes in psychological morbidities across the countries and territories. The results show Malaysia has the lowest prevalence of psychological morbidities depression (4.49\%), anxiety (4.36\%), stress $(5.10 \%)$, and insomnia (1.76\%) compared to other countries (Table 3). Some effective initiatives taken by the Malaysian Government reduced psychological illness among the population at the beginning of the pandemic such as increased the capacity of the hospital, isolation center, nationwide laboratories, allocated a huge budget for financial support, and ensured circulation of authentic information (Azlan et al., 2020; Kalok et al., 2020). People in Pakistan were more depressed (45\%) and anxious (34\%), Indians were highly stressed (40\%) and Italian were more sleepless $(29.76 \%)$ (Table 3). A study found that the poor sanitation, lack of basic preventive measures, lack of proper testing, and medical facilities are the reasons behind the higher psychological disorder, COVID-19 cases, and deaths in those countries (Wang et al., 2021).

Our results also show that the Eastern Mediterranean Region, African Region, Southeast Asia Region, and European Region are respectively most vulnerable in terms of the prevalence of depression (44.90\%), anxiety (49.60\%), stress $(40.49 \%)$, and insomnia (31.18\%) (Table 3).

Devastating scenarios such as poor food accessibility, lack of safe shelter, losing employment in several countries led to a higher likelihood of depression (Moradi, 2020). The prolonged period of isolation, poorer life quality, limited mobility, unstable treatment, and financial condition may lead the higher stress among the general population in the Southeast Asia region (Gopal et al., 2020; Kazmi et al., 2020). The literature shows that the history of medical issues, longer quarantine, and financial and health uncertainty were also the reasons for higher stress and insomnia during the pandemic (Agberotimi et al. 2020; Sigdel et al. 2020).

The prevalence of psychological morbidities also varies with different assessment tools. The highest pooled prevalence of depression of $29.10 \%$, anxiety of $31.93 \%$, stress of $37.30 \%$, and insomnia of $26.35 \%$ was for PHQ-9, DASS-21, PSS, and PSQL, respectively (Fig. 6). A meta-analysis and sytemetic review (Mahmud et al., 2021a) showed that HADS, HADS, PSS, and ISI respectively provide the highest prevalence of depression of $47.02 \%$, anxiety of $58.06 \%$, 
stress of $69.46 \%$, and insomnia of $46.58 \%$ among health care workers during the pandemic.

To our knowledge, this study is the most comprehensive systematic review and meta-analysis in investigating mental health difficulties among the general population during the COVID-19 pandemic. While other systematic reviews and meta-analyses were performed on some of the psychological morbidities, this study stands out in its use of comprehensive searches on four psychological issues, namely depression, anxiety, stress, and insomnia. This study also found out a large number of articles from all over the world, but other studies covered only two or three territories of the world. Besides, for the first time, we have observed periodic changes in the prevalence of depression, anxiety, stress, as well as insomnia by conducting subgroup analysis based on study durations.

These types of studies are inevitable for supporting public health globally and reducing the knowledge gap in the care of mental health disorders (Javadi et al., 2017). The funding bodies and governments can use this study as a tool to ensure sustainable development in mental health by supporting the prioritization and allocation of funds for mental health.

This study has some limitations. All of the studies included in this meta-analysis are cross-sectional and some of them are preprints. There are several assessment methods and cutoff points that were utilized for the same population screening in several studies. Even different cutoff points were considered for the same test in different studies. The prevalence estimates for some of the groups in subgroup analysis for depression, anxiety, stress, and insomnia based on both periods and assessment methods are estimated from few studies. In addition, there is a weak side to the omission of non-English papers and the exclusion of studies with low or moderate quality.

In conclusion, this systematic review provides a timely analysis of existing pieces of evidence that demonstrates a high prevalence of depression, anxiety, stress, and insomnia compared to normal time. If goes unnoticed for a long time, in severe cases, people may develop suicidal and self-destructive tendencies. This illustrates the significance of early detection and intervention for mental health problems in general people during the COVID-19 pandemic. The findings from subgroup analysis indicate that the prevalence of all four clinical symptoms is downward among the general population. However, this trend might change depending on the way the pandemic evolves. If the pandemic stays for a long time, the mental health of people might worsen further due to confinement, fear of infection, disruption in studies, financial crises, etc. Also, the prevalence might be different in individuals or different communities. Now, it is crucial to identify the most vulnerable group or individuals with psychological disorders from the general population. It is necessary to ascertain a holistic action plan to ensure strong mental health. In addition to medical facilities, psychological resources should also be established, adopted, and sustained. And thus, government and policymakers can apply the established strategies and interventions to prevent psychological adversities and enhance overall mental health in the general population. 
Author Contribution SM and AM conducted the searches. They also completed the screening text, extraction, and analysis of the data with the input from ND. MM and SM wrote the first draft of the manuscript with input from ND. ND, AM, and MM provided critical feedback. All authors discussed the results and contributed to the final manuscript.

Data Availability Datasets are available through the corresponding author upon reasonable request.

Materials and Code Availability Materials and Code are also available through the corresponding author.

\section{Declarations}

Ethics Approval Not applicable.

Consent to Participate Not applicable.

Consent for Publication Not applicable.

Conflict of Interest The authors declare no competing interests.

\section{References}

Agberotimi, S. F., Akinsola, O. S., Oguntayo, R., \& Olaseni, A. O. (2020). Interaction between socioeconomic status and mental health outcomes in the Nigerian context amid COVID-19 pandemic: A comparative study.

Akin, A., \& Çetın, B. (2007). The Depression Anxiety and Stress Scale (DASS): The study of validity and reliability. Educational Sciences: Theory \& Practice, 7(1).

Andersen, L. S., Grimsrud, A., Myer, L., Williams, D. R., Stein, D. J., \& Seedat, S. (2011). The psychometric properties of the K10 and K6 scales in screening for mood and anxiety disorders in the South African Stress and Health study. International Journal of Methods in Psychiatric Research, 20(4), 215-223.

Azlan, A. A., Hamzah, M. R., Sern, T. J., Ayub, S. H., \& Mohamad, E. (2020). Public knowledge, attitudes and practices towards COVID-19: A cross-sectional study in Malaysia. Plos One, 15(5), p.e0233668.

Bao, Y., Sun, Y., Meng, S., Shi, J., \& Lin, Lu. (2020). 2019-nCoV epidemic: Address mental health care to empower society. The Lancet, 395, e37-e38.

Bastien, C. H., Vallières, A., \& Morin, C. M. (2001). Validation of the Insomnia Severity Index as an outcome measure for insomnia research. Sleep Medicine, 2, 297-307.

Beck, A. T., Steer, R. A., \& Brown, G. Beck depression inventory-II. Psychological Assessment (1996).

Biggs, J. T., Wylie, L. T., \& Ziegler, V. E. (1978). Validity of the Zung self-rating depression scale. The British Journal of Psychiatry, 132(4), 381-385.

Brooks, S. K., Webster, R. K., Smith, L. E., Woodland, L., Wessely, S., Greenberg, N., Rubin G. J. (2020). The psychological impact of quarantine and how to reduce it: Rapid review of the evidence, The lancet.

Bryant, R. A., Moulds, M. L., \& Guthrie, R. M. (2000). Acute Stress Disorder Scale: A self-report measure of acute stress disorder. Psychological Assessment, 12(1), 61.

Buysse, D. J., Reynolds, C. F., 3rd., Monk, T. H., et al. (1989). The Pittsburgh Sleep Quality Index: A new instrument for psychiatric practice and research. Psychiatry Research, 28, 193-213.

Chen, S., \& Bonanno, G. A. (2020). Psychological adjustment during the global outbreak of COVID-19: A resilience perspective. Psychological Trauma: Theory, Research, Practice, and Policy, 12(S1), S51.

Chen, Q., Liang, M., Li, Y., Guo, J., Fei, D., Wang, L., He, Li., Sheng, C., Cai, Y., \& Li, X. (2020). Mental health care for medical staff in China during the COVID-19 outbreak. The Lancet Psychiatry, 7 , e15-e16. 
Chua, S. E., Cheung, V., McAlonan, G. M., Cheung, C., Wong, J. W., Cheung, E. P., Chan, M. T., Wong, T. K., Choy, K. M., Chu, C. M., \& Lee, P. W. (2004). Stress and psychological impact on SARS patients during the outbreak. The Canadian Journal of Psychiatry, 49(6), 385-390.

Cooke, J. E., Eirich, R., Racine, N., \& Madigan, S. (2020). Prevalence of posttraumatic and general psychological stress during COVID-19: A rapid review and meta-analysis, Psychiatry Research: 113347.

Dai, H., Zhang, S. X., Looi, K. H., Rui, Su., \& Li, J. (2020). Perception of health conditions and test availability as predictors of adults' mental health during the COVID-19 pandemic: A survey study of adults in Malaysia. International Journal of Environmental Research and Public Health, 17, 5498.

Derogatis, L. R., \& Cleary, P. A. (1977). Confirmation of the dimensional structure of the SCL-90: A study in construct validation. Journal of Clinical Psychology, 33, 981-989.

Egger, M., Smith, G. D., Schneider, M., \& Minder, C. (1997). Bias in meta-analysis detected by a simple, graphical test. BMJ, 315, 629-634.

Fu, W., Wang, C., Zou, Li., Guo, Y., Zuxun, Lu., Yan, S., \& Mao, J. (2020). Psychological health, sleep quality, and coping styles to stress facing the COVID-19 in Wuhan. China, Translational Psychiatry, 10, 1-9.

Fydrich, T., Dowdall, D., \& Chambless, D. L. (1992). Reliability and validity of the Beck Anxiety Inventory. Journal of Anxiety Disorders, 6(1), 55-61.

Gao, J., Zheng, P., Jia, Y., Chen, H., Mao, Y., Chen, S., Wang, Y., Fu, H., \& Dai, J. (2020). Mental health problems and social media exposure during COVID-19 outbreak. PLoS ONE, 15(4), e0231924. https://doi.org/10.1371/journal.pone.0231924

Gopal, A., Sharma, A. J., \& Subramanyam, M. A. (2020). Dynamics of psychological responses to COVID-19 in India: A longitudinal study. PloS One, 15(10), p.e0240650.

Goulia, P., Mantas, C., Dimitroula, D., Mantis, D., \& Hyphantis, T. (2010). General hospital staff worries, perceived sufficiency of information and associated psychological distress during the A/H1N1 influenza pandemic. BMC Infectious Diseases, 10(1), 322.

Grover, S., Sahoo, S., Mehra, A., Avasthi, A., Tripathi, A., Subramanyan, A., Amrit Pattojoshi, G., Rao, P., Saha, G., \& Mishra, K. K. (2020). Psychological impact of COVID-19 lockdown: An online survey from India. Indian Journal of Psychiatry, 62, 354.

Gualano, M. R., Moro, G. L., Voglino, G., Bert, F., \& Siliquini, R. (2020). Effects of Covid-19 lockdown on mental health and sleep disturbances in Italy. International Journal of Environmental Research and Public Health, 17, 4779.

Hann, D., Winter, K., \& Jacobsen, P. (1999). Measurement of depressive symptoms in cancer patients: Evaluation of the Center for Epidemiological Studies Depression Scale (CES-D). Journal of Psychosomatic Research, 46(5), 437-443.

Huang, Y., Wang, Y., Wang, H., Liu, Z., Yu, X., Yan, J., Yu, Y., Kou, C., Xu, X., Lu, J., \& Wang, Z. (2019). Prevalence of mental disorders in China: A cross-sectional epidemiological study. The Lancet Psychiatry, 6(3), 211-224.

Huang, C., Wang, Y., Li, X., Ren, L., Zhao, J., Yi, Hu., Zhang, Li., Fan, G., Jiuyang, Xu., \& Xiaoying, Gu. (2020). Clinical features of patients infected with 2019 novel coronavirus in Wuhan. China, the Lancet, 395, 497-506.

Huang, Y., \& Zhao, N. (2020). Mental health burden for the public affected by the COVID-19 outbreak in China: Who will be the high-risk group? Psychology, Health \& Medicine: 1-12.

Islam, Md A., Barna, S. D., Raihan, H., Khan, Md N. A., \& Hossain, Md T. (2020). Depression and anxiety among university students during the COVID-19 pandemic in Bangladesh: A web-based crosssectional survey, PLoS One, 15: e0238162.

Javadi, D., Feldhaus, I., Mancuso, A., \& Ghaffar, A. (2017). Applying systems thinking to task shifting for mental health using lay providers: a review of the evidence, Global Mental Health, 4.

Jeong, H., Yim, H. W., Song, Y. J., Ki, M., Min, J. A., Cho, J., \& Chae, J. H. (2016). Mental health status of people isolated due to Middle East respiratory syndrome. Epidemiology and Health, 38.

Kalok, A., Sharip, S., Abdul Hafizz, A. M., Zainuddin, Z. M., \& Shafiee, M. N. (2020). The psychological impact of movement restriction during the COVID-19 outbreak on clinical undergraduates: A cross-sectional study. International Journal of Environmental Research and Public Health, 17(22), 8522 .

Kazmi, S. S. H., Hasan, K., Talib, S., \& Saxena, S. (2020). COVID-19 and lockdwon: A study on the impact on mental health, Available at SSRN 3577515. 
Keita, M.M., Taverne, B., Savané, S.S., March, L., Doukoure, M., Sow, M.S., Touré, A., Etard, J.F., Barry, M., Delaporte, E. and PostEboGui Study Group. (2017). Depressive symptoms among survivors of Ebola virus disease in Conakry (Guinea): Preliminary results of the PostEboGui cohort. BMC Psychiatry, 17(1), 127.

Krishnamoorthy, Y., Nagarajan, R., Saya, G.K. \& Menon, V., 2020. Prevalence of psychological morbidities among general population, healthcare workers and COVID-19 patients amidst the COVID-19 pandemic: A systematic review and meta-analysis. Psychiatry Research, 293, p.113382.

Kroenke, K., Spitzer, R. L., \& Williams, J. B. W (2003). The Patient Health Questionnaire-2: Validity of a two-item depression screener. Medical care, 1284-1292.

Lee, E. .-H. . (2012). Review of the Psychometric Evidence of the Perceived Stress Scale. Asian Nursing Research, 6(4), 121-127. https://doi.org/10.1016/j.anr.2012.08.004.

Liberati, A., Altman, D. G., Tetzlaff, J., \& Mulrow, C. (2009). The PRISMA statement for reporting systematic reviews and metaanalyses of studies that evaluate health care interventions: Explanation and elaboration. Journal of Clinical Epidemiology, 62, e1-34.

Lim, G. Y., Tam, W. W., Lu, Y., Ho, C. S., Zhang, M. W., \& Ho, R. C. (2018). Prevalence of depression in the community from 30 countries between 1994 and 2014. Science and Reports, 8(1), 2861. https://doi.org/10.1038/s41598-018-21243-X

Liu, D., Ren, Y., Yan, F., Li Y., Xu, X., Yu, X., Qu, W., Wang, Z., Tian, B., \& Yang, F. (2020). Psychological impact and predisposing factors of the coronavirus disease 2019 (COVID-19) pandemic on general public in China.

Magán, I., Sanz, J., \& García-Vera, M. P. (2008). Psychometric properties of a Spanish version of the Beck Anxiety Inventory (BAI) in general population. The Spanish Journal of Psychology, 11(2), 626.

Mahmud, S., Hossain, S., Muyeed, A., Islam, M. M., \& Mohsin, M. (2021a). The global prevalence of depression, anxiety, stress, and, insomnia and its' changes among health professionals during COVID-19 pandemic: a rapid systematic review and meta-analysis. Heliyon, p.e07393.

Mahmud, S., Mohsin, M., Hossain, S., Islam, M. M., \& Muyeed, A. (2021b). The acceptance of COVID19 vaccine: a global rapid systematic review and meta-analysis. Available at SSRN 3855987.

Mahmud, S., Mohsin, M., Khan, I. A., Mian, A. U., \& Zaman, M. A. (2021c). Knowledge, beliefs, attitudes and perceived risk about COVID-19 vaccine and determinants of COVID-19 vaccine acceptance in Bangladesh. PLOS ONE, 16(9), e0257096. https://doi.org/10.1371/journal.pone.0257096

Marelli, S., Castelnuovo, A., Somma, A., Castronovo, V., Mombelli, S., Bottoni, D., Leitner, C., Fossati, A., \& Ferini-Strambi, L. (2020). Impact of COVID-19 lockdown on sleep quality in university students and administration staff, Journal of Neurology: 1-8.

Mazza, C., Ricci, E., Biondi, S., Colasanti, M., Ferracuti, S., Napoli, C., \& Roma, P. (2020). A nationwide survey of psychological distress among Italian people during the COVID-19 pandemic: Immediate psychological responses and associated factors. International Journal of Environmental Research and Public Health, 17, 3165.

Mc Cracken, L. M., Badinlou, F., Buhrman, M., \& Brocki, K. C. (2020). Psychological impact of COVID-19 in the Sweden Population: Depression, Anxiety, and Insomnia and their Associations to Risk and Vulnerability factors, European Psychiatry: 1-30.

Meng, H., Xu, Y., Dai, J., Zhang, Y., Liu, B., \& Yang, H. (2020). Analyze the psychological impact of COVID-19 among the elderly population in China and make corresponding suggestions, Psychiatry Research, 289: 112983.

Moradi, L. (2020). COVID-19 in Eastern Mediterranean region countries. Journal of Archives in Military Medicine, 8(4)

O’Connor, D. B., Aggleton, J. P., Chakrabarti, B., Cooper, C. L., Creswell, C., Dunsmuir, S., Fiske, S. T., Gathercole, S., Gough, B., Ireland, J. L., \& Jones, M. V. (2020). Research priorities for the COVID19 pandemic and beyond: A call to action for psychological science.

Odriozola-González, P., Planchuelo-Gómez, Á., Irurtia-Muñiz, M. J., \& de Luis-García, R. (2020). Psychological symptoms of the outbreak of the COVID-19 crisis and confinement in the population of Spain.

Othman, N. (2020). Depression, anxiety, and stress in the time of COVID-19 pandemic in Kurdistan Region, Iraq, Kurdistan. Journal of Applied Research: 37-44.

Ozamiz-Etxebarria, N., Dosil-Santamaria, M., Picaza-Gorrochategui, M., \& Idoiaga-Mondragon, N. (2020). Stress, anxiety, and depression levels in the initial stage of the COVID-19 outbreak in a population sample in the northern Spain, Cadernos de Saúde Pública, 36: e00054020. 
Pan, K. Y., Kok, A. A., Eikelenboom, M., Horsfall, M., Jörg, F., Luteijn, R. A., Rhebergen, D., van Oppen, P., Giltay, E. J., \& Penninx, B. W. (2020). The mental health impact of the COVID-19 pandemic on people with and without depressive, anxiety, or obsessive-compulsive disorders: A longitudinal study of three Dutch case-control cohorts. The Lancet Psychiatry.

Pappa, S., Ntella, V., Giannakas, T., Giannakoulis, V. G., Papoutsi, E., \& Katsaounou, P. (2020). Prevalence of depression, anxiety, and insomnia among healthcare workers during the COVID-19 pandemic: A systematic review and meta-analysis. Brain, Behavior, and Immunity.

Pieh, C., Budimir, S., \& Probst, T. (2020). The effect of age, gender, income, work, and physical activity on mental health during coronavirus disease (COVID-19) lockdown in Austria. Journal of Psychosomatic Research, 136: 110186.

Qiu, W., Rutherford, S., Mao, A., \& Chu, C. (2017). The pandemic and its impacts. Health, Culture and Society, 9, 1-11.

Radloff, L. S. (1977). The CES-D scale: A self-report depression scale for research in the general population. Applied Psychological Measurement, 1(3), 385-401.

Rossi, R., Socci, V., Talevi, D., Mensi, S., Niolu, C., Pacitti, F., Di Marco, A., Rossi, A., Siracusano, A., \& Di Lorenzo, G. (2020). COVID-19 pandemic and lockdown measures impact on mental health among the general population in Italy. Frontiers in Psychiatry, 11.

Ryu, S., Chun, B. C., \& Korean Society Epidemiology. (2020). An interim review of the epidemiological characteristics of 2019 novel coronavirus. Epidemiology and Health, 42.

Salman, M., Asif, N., Ul Mustafa, Z., Khan, T. M., Shehzadi, N., Hussain, K., Tahir, H., Raza, M. H., \& Tanveer Khan, M. (2020). Psychological impact of COVID-19 on Pakistani University students and how they are coping, medRxiv.

Salari, N., Hosseinian-Far, A., Jalali, R., Vaisi-Raygani, A., Rasoulpoor, S., Mohammadi, M., Rasoulpoor, S., \& Khaledi-Paveh, B. (2020). Prevalence of stress, anxiety, depression among the general population during the COVID-19 pandemic: A systematic review and meta-analysis. Globalization and Health, 16, 1-11.

Shevlin, M., Nolan, E., Owczarek M., McBride, O., Murphy, J., Gibson Miller, J., \& McKay, R. (2020). COVID-19-related anxiety predicts somatic symptoms in the UK population. British Journal of Health Psychology.

Shi, Le., Zheng-An, Lu., Que, J.-Y., Huang, X.-L., Liu, L., Ran, M.-S., Gong, Y.-M., Yuan, K., Yan, W., $\&$ Sun, Y.-K. (2020). Prevalence of and risk factors associated with mental health symptoms among the general population in China during the coronavirus disease 2019 pandemic. JAMA Network Open, 3, e2014053-e2014153.

Sigdel, A., Bista, A., Bhattarai, N., Chandra Poon, B., Giri, G., \& Marqusee, H. (2020). Depression, anxiety and depression-anxiety comorbidity amid COVID-19 pandemic: An online survey conducted during lockdown in Nepal, medRxiv.

da Silva, F. C. T., \& Neto, M. L. R. (2020). Psychiatric symptomatology associated with depression, anxiety, distress, and insomnia in health professionals working in patients affected by COVID-19: A systematic review with meta-analysis, Progress in Neuro-Psychopharmacology and Biological Psychiatry: 110057.

Soldatos, C. R., Dikeos, D. G., \& Paparrigopoulos, T. J. (2003). The diagnostic validity of the Athens Insomnia Scale. Journal of Psychosomatic Research, 55(3), 263-267.

Song, L., Wang, Y., Li, ZhengLin, Yang, Y., \& Li, H. (2020). Mental health and work attitudes among people resuming work during the COVID-19 pandemic: A cross-sectional study in China. International Journal of Environmental Research and Public Health, 17, 5059.

Spitzer, R. L., Kroenke, K., Williams, J. B. W., et al. (2006). A brief measure for assessing generalized anxiety disorder: The GAD-7. Archives of Internal Medicine, 166, 1092-1097.

Tang, F., Liang, J., Zhang, H., Kelifa, M. M., He, Q., \& Wang, P. (2020). COVID-19 related depression and anxiety among quarantined respondents. Psychology \& Health: 1-15.

Ueda, M., Stickley, A., Sueki, H., \& Matsubayashi, T. (2020). Mental health status of the general population during the COVID-19 pandemic: A cross-sectional national survey in Japan, medRxiv.

Verma, S., \& Mishra, A. (2020). Depression, anxiety, and stress and socio-demographic correlates among general Indian public during COVID-19. International Journal of Social Psychiatry: 0020764020934508.

Wang, C., Pan, R., Wan, X., Tan, Y., Linkang, Xu., Ho, C. S., \& Ho, R. C. (2020a). Immediate psychological responses and associated factors during the initial stage of the 2019 coronavirus disease (COVID-19) epidemic among the general population in China. International Journal of Environmental Research and Public Health, 17, 1729. 
Wang, S., Zhang, Y., Ding, W., Meng, Y., Hu, H., Liu, Z., Zeng, X., \& Wang, M. (2020b). Psychological distress and sleep problems when people are under interpersonal isolation during an epidemic: A nationwide multicenter cross-sectional study. European Psychiatry, 63.

Wang, Y., Di, Y., Ye, J., \& Wei, W. (2020c). Study on the public psychological states and its related factors during the outbreak of coronavirus disease 2019 (COVID-19) in some regions of China, Psychology. Health \& Medicine: 1-10.

Wang, Y., Kala, M. P., \& Jafar, T.H. (2020d). Factors associated with psychological distress during the coronavirus disease 2019 (COVID-19) pandemic on the predominantly general population: A systematic review and meta-analysis. PloS One, 15(12), p.e0244630.

Wang, C., Tee, M., Roy, A. E., Fardin, M. A., Srichokchatchawan, W., Habib, H. A., Tran, B. X., Hussain, S., Hoang, M. T., Le, X. T., \& Ma, W. (2021). The impact of COVID-19 pandemic on physical and mental health of Asians: A study of seven middle-income countries in Asia. PloS One, 16(2), p.e0246824.

Wells, A. (2005). Generalized anxiety disorder. In Encyclopedia of cognitive behavior therapy (pp. 195198). Springer.

World Health Organization (n.d.) | Countries, https://www.who.int/countries. Accessed 2 July 2021

Xiong, J., Lipsitz, O., Nasri, F., Lui, L. M., Gill, H., Phan, L., Chen-Li, D., Iacobucci, M., Ho, R., Majeed, A., McIntyre, R. S. Impact of COVID-19 pandemic on mental health in the general population: A systematic review. Journal of Affective Disorders. 2020 Aug 8.

Zhou, S.-J., Wang, L.-L., Yang, R., Yang, X.-J., Zhang, L.-G., Guo, Z.-C., Chen, J.-C., Wang, J.-Q., \& Chen, J.-X. (2020a). Sleep problems among Chinese adolescents and young adults during the coronavirus-2019 pandemic. Sleep Medicine.

Zhou, Shuang-Jiang, Li-Gang Zhang, Lei-Lei Wang, Zhao-Chang Guo, Jing-Qi Wang, Jin-Cheng Chen, Mei Liu, Xi Chen, and Jing-Xu Chen. (2020b). Prevalence and socio-demographic correlates of psychological health problems in Chinese adolescents during the outbreak of COVID-19. European Child \& Adolescent Psychiatry: 1-10.

Zung, W.W. (1965). A self-rating depression scale. Arch Gen Psychiatry. 